\title{
TRAF6 regulates satellite stem cell self-renewal and function during regenerative myogenesis
}

\author{
Sajedah M. Hindi and Ashok Kumar \\ Department of Anatomical Sciences and Neurobiology, University of Louisville School of Medicine, Louisville, Kentucky, USA.
}

\begin{abstract}
Satellite cells are a stem cell population within adult muscle and are responsible for myofiber regeneration upon injury. Satellite cell dysfunction has been shown to underlie the loss of skeletal muscle mass in many acquired and genetic muscle disorders. The transcription factor paired box-protein-7 (PAX7) is indispensable for supplementing the reservoir of satellite cells and driving regeneration in normal and diseased muscle. TNF receptor-associated factor 6 (TRAF6) is an adaptor protein and an $\mathrm{E} 3$ ubiquitin ligase that mediates the activation of multiple cell signaling pathways in a context-dependent manner. Here, we demonstrated that TRAF6-mediated signaling is critical for homeostasis of satellite cells and their function during regenerative myogenesis. Selective deletion of Traf6 in satellite cells of adult mice led to profound muscle regeneration defects and dramatically reduced levels of PAX7 and late myogenesis markers. TRAF6 was required for the activation of MAPKs ERK1/2 and JNK1/2, which in turn activated the transcription factor c-JUN, which binds the Pax7 promoter and augments Pax7 expression. Moreover, TRAF6/c-JUN signaling repressed the levels of the microRNAs miR-1 and miR-206, which promote differentiation, to maintain PAX7 levels in satellite cells. We also determined that satellite cell-specific deletion of Traf6 exaggerates the dystrophic phenotype in the mdx (a mouse model of Duchenne muscular dystrophy) mouse by blunting the regeneration of injured myofibers. Collectively, our study reveals an essential role for TRAF6 in satellite stem cell function.
\end{abstract}

\section{Introduction}

Satellite cells are stem cells within skeletal muscle, and they reside between the plasma membrane and basal lamina in a quiescent state characterized by low-metabolic activity and reversible mitotic arrest. These cells are primarily responsible for growth, maintenance, and repair of injured myofibers in adults $(1,2)$. In response to muscle injury, satellite cells are rapidly activated to reenter the cell cycle, undergo several rounds of proliferation, and then differentiate into myoblasts, which eventually fuse with each other or preexisting myofibers to complete the repair process $(3,4)$. While most of the activated satellite cells differentiate into myogenic lineage, a small portion of them self-renews and returns to quiescence to respond to the next round of muscle injury and repair $(3,4)$.

Transcription factor paired box-protein-7 (PAX7) is a critical regulator for satellite cell biogenesis, survival, specification, and self-renewal $(5,6)$. Quiescent satellite cells express high levels of PAX7, whereas other myogenic regulatory factors (MRFs) such as MYF5 and MyoD proteins are undetectable. In proliferating satellite cells, PAX7 persists at lower levels, whereas the levels of PAX7 are completely repressed in myogenic lineage cells that commit to terminal differentiation $(3,7,8)$. The critical role of $\mathrm{PAX} 7$ in maintaining satellite cell quiescence or self-renewal is also evident by the findings that forced expression of PAX7 inhibits myogenesis and cell cycle progression in satellite cells, result-

Conflict of interest: The authors have declared that no conflict of interest exists. Submitted: September 14, 2015; Accepted: October 16, 2015.

Reference information: J Clin Invest. 2016;126(1):151-168. doi:10.1172/JCI81655 ing in the maintenance of their inactivated state (9). Genetic deletion of PAX7 in satellite cells of adult mice led to failure of myofiber regeneration due to precocious differentiation (10). However, the molecular and signaling mechanisms that regulate the levels of PAX7 and satellite cell self-renewal and differentiation remain poorly understood.

TRAF6 is a crucial adaptor protein that mediates signaling events from TNF receptor superfamily, interleukin-1 receptor (IL-1R) family, and TLR family (11). TRAF6 is also a nonconventional RING finger E3 ligase, which catalyzes formation of K63linked ubiquitin chains $(12,13)$ and activates multiple signaling pathways (11-14). TRAF6 plays critical roles in innate immune response and regulates the function of antigen-presenting cells $(14,15)$. TRAF6-deficient mice die between 2-3 weeks due to severe osteopetrosis $(16,17)$. Furthermore, TRAF6 plays a crucial role in lymph node organogenesis, formation of skin appendices, and development of nervous system (14).

TRAF6 is highly expressed in cultured myogenic cells, and its levels are reduced upon differentiation of myoblasts into myotubes. Similarly, TRAF6 is expressed at high levels during the early stages of muscle development, and its levels gradually subside during development to the adult stage (18). Intriguingly, the levels of TRAF6 and its E3 ubiquitin ligase activity are considerably increased in skeletal muscle of adult mice in many catabolic conditions $(18,19)$. Myofiber-specific deletion of Traf6 inhibits muscle wasting in response to denervation, cancer cachexia, or starvation through distinct mechanisms $(18,19)$. Levels of TRAF6 are also increased in skeletal muscle of WT mice in response to acute injury (20) and in dystrophic muscle of mdx (a mouse model of Duch- 
A
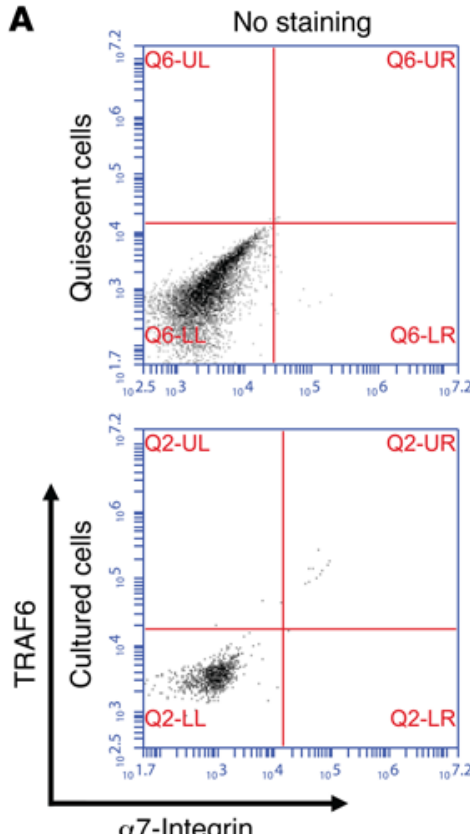

$\alpha 7$-Integrin
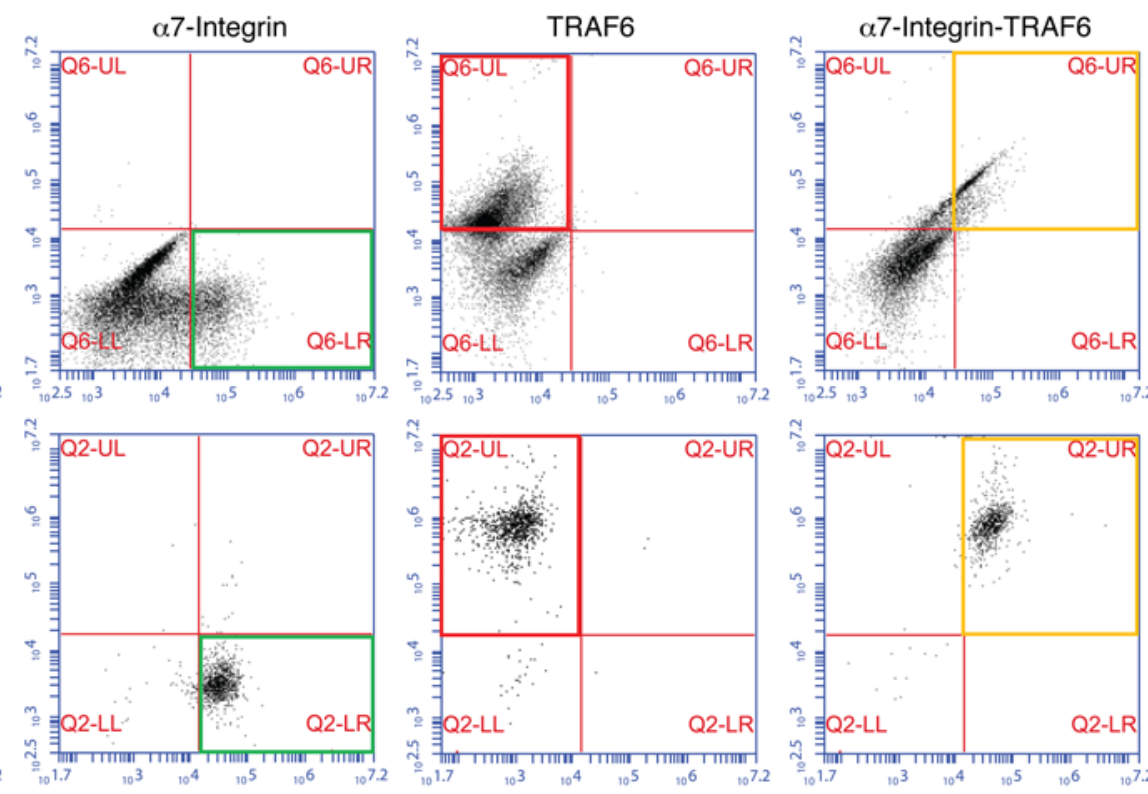

B

Anti-TRAF6

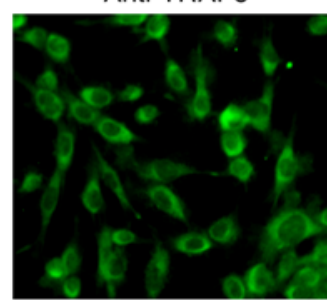

Anti-PAX7

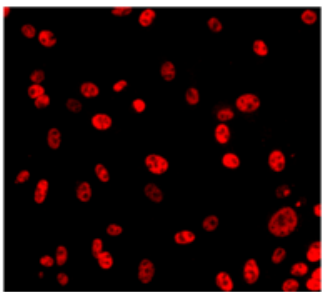

DAPI

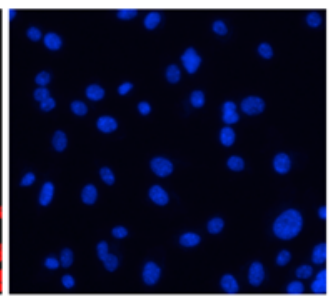

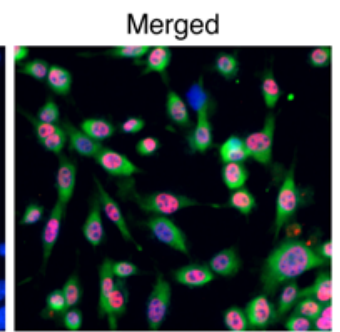

C Time in DM (h)

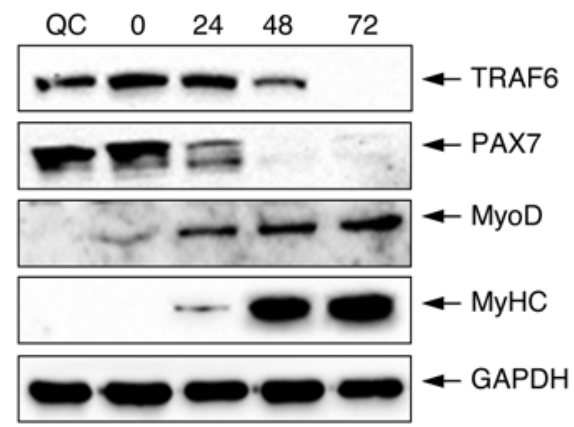

Figure 1. Expression of TRAF6 in satellite cells. Primary mononucleated cells were isolated from hind limb muscle of WT mice and subjected for FACS analysis for the expression of $\alpha$ 7-integrin and TRAF6, either immediately after isolation (quiescent) or after purification and culturing (activated). (A) Representative dot plots from 4 independent experiments displaying enrichment of TRAF6+ cells among $\alpha 7$-intigrin ${ }^{+}$population in both freshly isolated and cultured myoblasts. (B) Primary myogenic cells were immunostained for TRAF6 and PAX7 proteins. The nuclei were labeled using DAPI. Representative photomicrographs from 3 replicates show colocalization of TRAF6 and PAX7 in mouse primary myoblasts. Original magnification, $\times 20$. (C) Freshly sorted satellite cells were either frozen at isolation (QC, quiescent cells) or seeded and cultured in DM for various time points. Immunoblots demonstrate the levels of TRAF6, PAX7, MyoD, MyHC, and unrelated protein GAPDH in QC and in cultured primary myoblasts at different time points after addition of DM.

enne muscular dystrophy) mice (21). Skeletal muscle-specific deletion of Traf6 improves regeneration of injured myofibers potentially through limiting the inflammatory milieu and augmenting Notch signaling, which increases the proliferation of satellite cells in a non-cell-autonomous manner $(20,21)$. Interestingly, we found that myofiber-specific deletion of Traf6 causes only partial reduc- tion in its protein levels in injured muscle, suggesting that other cell types are also responsible for the elevated levels of TRAF6 in injured muscle microenvironment (20). Recently, it has been reported that TRAF6 is required for the differentiation of cultured myoblasts $(22,23)$. However, the role of TRAF6 in homeostasis and function of satellite stem cells has not been yet investigated. 
In this study, using an inducible conditional knockout approach, we demonstrate that TRAF6 is critical for the expression of PAX7, maintenance of the satellite stem cell pool, and regeneration of injured skeletal muscle upon injury. Our results demonstrate that TRAF6 signaling is required for the activation of ERK1/2 and JNK1/2 MAPKs, which causes the phosphorylation of transcription factor c-JUN/AP1 (activator protein 1) that in turn binds to the Pax7 promoter to augment the gene expression of PAX7. TRAF6-mediated signaling also prevents c-JUN from undergoing proteolytic degradation in satellite cells. The critical role of TRAF6 in regenerative myogenesis is also supported by our findings that targeted deletion of TRAF6 in satellite cells of mdx mice exacerbates dystrophic phenotype due to impairment in myofiber regeneration. Furthermore, our results show that TRAF6-deficient satellite cells have poor engraftment potential when transplanted into dystrophic muscle of mdx mice.

\section{Results}

TRAF6 is expressed in satellite cells of adult mice. We first studied whether satellite cells express TRAF6. Due to the lack of a suitable antibody that can distinctly detect TRAF6 in satellite cells on muscle sections, we decided to use FACS approach. To understand whether TRAF6 is expressed in quiescent satellite cells, cell suspensions isolated from mouse hind limb skeletal muscle were directly FACS sorted for $\alpha$ 7-integrin (a marker for satellite cells) and TRAF6 after eliminating possible infiltrating cells $\left(\mathrm{CD} 45^{+}\right.$, CD $31^{+}, \mathrm{Sca}_{-1} 1^{+}$, and Ter119 ${ }^{+}$. The strategy used for FACS analysis and staining with control antibodies are presented in Supplemental Figure 1 (supplemental material available online with this article; doi:10.1172/JCI81655DS1). This analysis showed that $\alpha 7$-integrin $^{+}$cells were predominantly TRAF6 $6^{+}$(Figure 1A, upper panel). We next prepared primary myogenic cultures (after eliminating fibroblasts through serial preplating) and studied the expression of TRAF6. Results showed that TRAF6 was also coexpressed with $\alpha 7$-integrin in cultured primary myoblasts (Figure 1A, lower panel). PAX7 transcription factor is expressed in both activated and quiescent satellite cells $(5,6)$. Immunocytochemical analysis showed that TRAF6 was predominantly expressed in the cytoplasm and PAX7 was localized in the nucleus. Moreover, all $\mathrm{PAX} 7^{+}$cells were also TRAF6 ${ }^{+}$, further confirming that TRAF6 is expressed in satellite cells (Figure 1B).

We next isolated $\alpha 7$-integrin ${ }^{+}$and $\mathrm{CD}^{-} 5^{-}, \mathrm{CD} 31^{-}, \mathrm{Sca}-1^{-}$, and Ter119- cells from 8-week-old WT mice by FACS sorting technique. The cells were immediately frozen in liquid nitrogen, and the cell lysates made were probed for TRAF6, MyoD, and PAX7 levels. This analysis showed that TRAF6 and PAX7 are highly expressed in both quiescent and activated (cultured) satellite cells (Figure 1C). MyoD was detectable only in cultured myogenic cells - not in quiescent satellite cells - which is consistent with published reports that MyoD is expressed only in activated satellite cells $(1,24)$. We also examined the levels of TRAF6, PAX7, MyoD, and myosin heavy chain (MyHC, a marker of muscle differentiation) in primary myoblast cultures at different time points after incubation in differentiation medium (DM). Consistent with previous findings $(9,18,25)$, the levels of TRAF6 and PAX7 were gradually decreased, whereas MyoD and MyHC levels were increased upon incubation of cells in DM. Collectively, these results suggest that TRAF6 is expressed in both quiescent and activated satellite cells, and its levels are reduced during progression through the myogenic lineage.

TRAF6 signaling in satellite cells is essential for regeneration of injured myofibers in adult mice. To investigate the role of TRAF6 in satellite cell function, we crossed Traf $6^{f / f l}$ mice with tamoxifen inducible Pax7-Cre mice (Pax7-CreER mice) to generate satellite cell-specific Traf6 knockout (henceforth, TRAF6 ${ }^{\text {scko }}$ ) mice. Eightweek-old littermate $\operatorname{Traf}^{f / / f l}$ and TRAF $6^{\text {scko }}$ mice were i.p. injected with tamoxifen for 4 consecutive days and kept on tamoxifen-containing standard chow for the duration of the experiment (Supplemental Figure 2). Tibial anterior muscle (TA muscle) of adult $\operatorname{Traf}^{f / / f l}$ and TRAF6 $6^{\text {scko }}$ mice was injured through i.m. injection of $1.2 \% \mathrm{BaCl}_{2}$ solution. The muscle was isolated at 5,10 , and 21 days after injury and analyzed by performing H\&E staining. Strikingly, we found that muscle regeneration was dramatically reduced in TRAF6 $6^{\text {scko }}$ mice compared with littermate $\operatorname{Traf}^{f / / f l}$ mice (Figure 2A). At day 5 after $\mathrm{BaCl}_{2}$ injection, newly formed centronucleated fibers (CNF) populated regenerating TA muscle of Traf6 $6^{f / f l}$ mice. By contrast, TA muscle of corresponding TRAF $6^{\text {scko }}$ mice showed poor signs of regeneration, evident by the reduction in myofiber cross-sectional area (CSA) and number of CNF (Figure 2, A-D) and robust persistence of cellular infiltrate (Figure 2A). Manifestations of defective muscle repair in TRAF $6^{\text {scko }}$ mice were still evident at day 21 after muscle injury, where regenerated muscle of control $\operatorname{Traf}^{f / / f l}$ displayed complete subsidence of inflammatory cells and reconstitution of normal muscle (Figure 2A). Signs of impaired muscle regeneration in TRAF $6^{\text {scko }}$ mice were even more pronounced when TA muscle was subjected to a second round of injury using $\mathrm{BaCl}_{2}$ injection (Figure $2 \mathrm{E}$ ). Regenerating areas of TRAF6 ${ }^{\text {scko }}$ muscle were notably void of newly formed muscle fibers and displayed massive deposition of fibrotic and adipogenic tissue at day 5 after second injury (Figure 2F).

Ablation of TRAF6 in satellite cells impairs the regeneration program in injured myofibers. Hierarchical expression of MRFs MYF5, MyoD, and myogenin, as well as embryonic/developmental isoform of $\mathrm{MyHC}(\mathrm{eMyHC})$, is required for completing the myogenic program $(7,26)$. Our immunohistochemical analysis revealed that regenerating muscle from TRAF6 $6^{\text {scko }}$ mice contained drastically reduced number and smaller size $\mathrm{eMyHC}^{+}$fibers compared with corresponding $\operatorname{Traf}^{f / / f l}$ at day 3 and day 5 after injury (Figure 3, A-C). To understand whether deletion of TRAF6 in satellite cells delays or disrupts regeneration of skeletal muscle after injury, we performed Western blot to detect the levels of eMyHC at different time points after injury. There was a considerable increase in the levels of eMyHC protein in Traf $6^{f / f l}$ mice, which peaked at day 5 and 7. Interestingly, there was no such increase in TRAF6 ${ }^{\text {scko }}$ muscle even after 15 days of muscle injury, suggesting defects in regenerative myogenesis in TRAF6 ${ }^{\text {scko }}$ mice (Figure 3D). Moreover, the mRNA levels of eMyHC (i.e., Myh3), Myf5, Myod, and myogenin were found to be significantly reduced in injured TA muscle of TRAF ${ }^{\text {scko }}$ mice compared with Traf $6^{\text {fl/l }}$ (Figure 3, E and F). Intriguingly, mRNA levels of $M y f 5$ were also found to be significantly reduced in contralateral uninjured TA muscle of TRAF $6^{\text {scko }}$ mice compared with Traf $6^{f / f l}$ (Figure 3F), leading to the theory that TRAF6 has a pivotal role in regulating the early stages of myogenesis in satellite stem cells. 
A

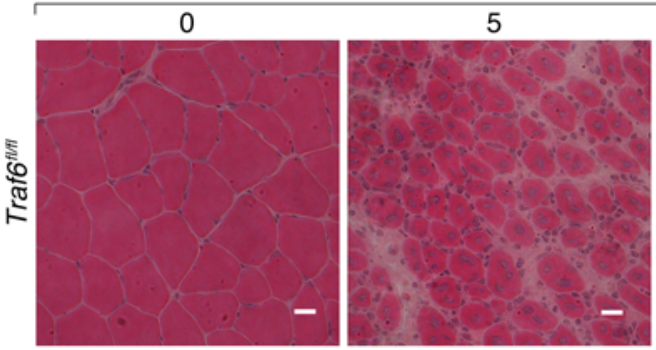

Time after injury (d)
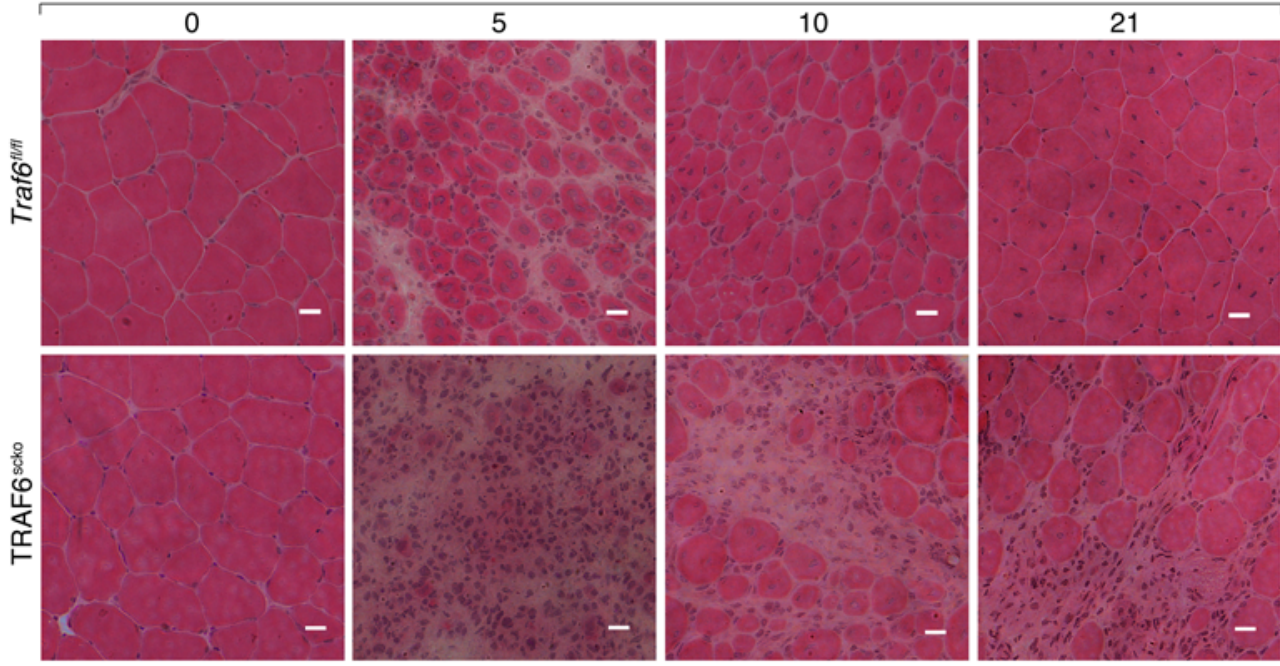

B

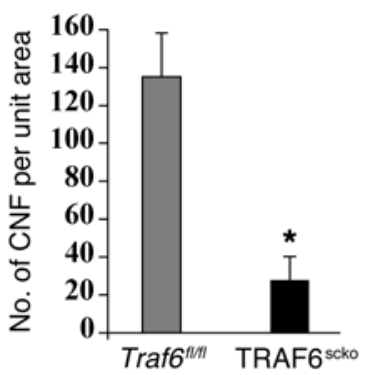

C

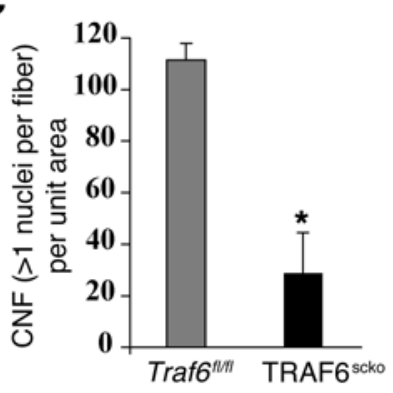

D

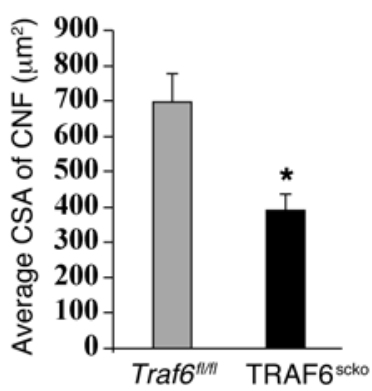

E



$\mathbf{F}$

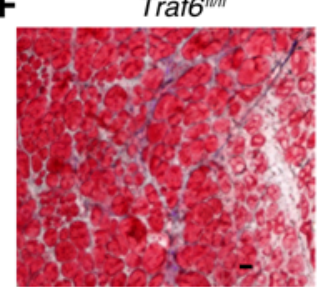

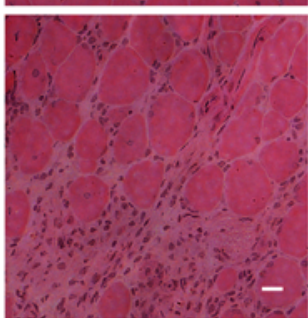

So 40
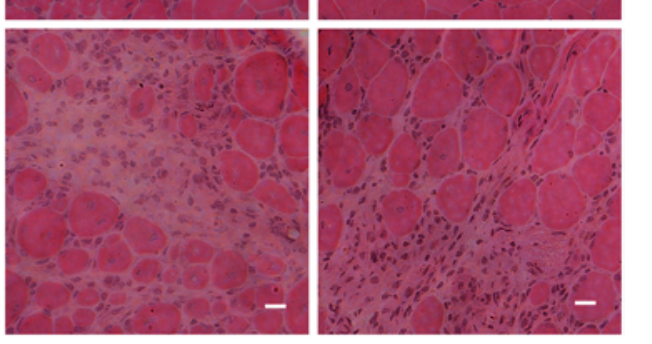

\section{政}


A

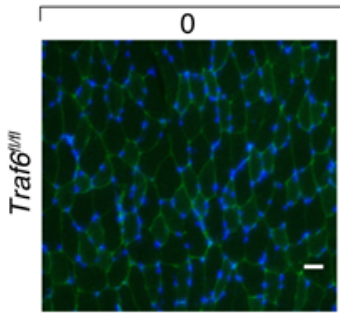

Time after injury (d)
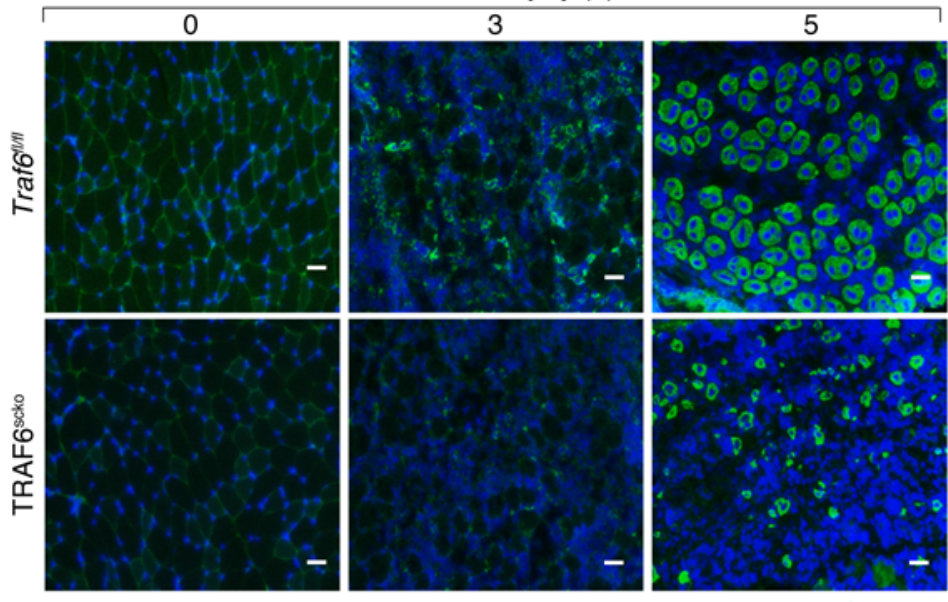

eMyHC; DAPI
B

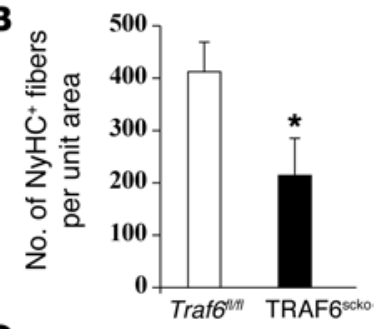

C

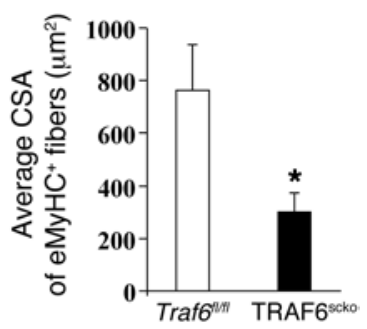

D

Traf6"III

TRAF6 $6^{\text {scko }}$

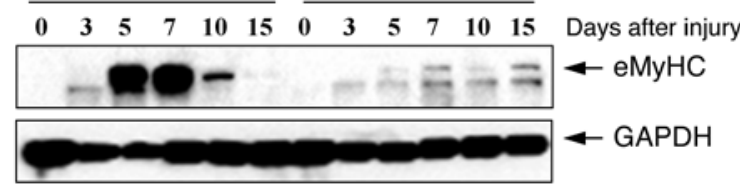

E

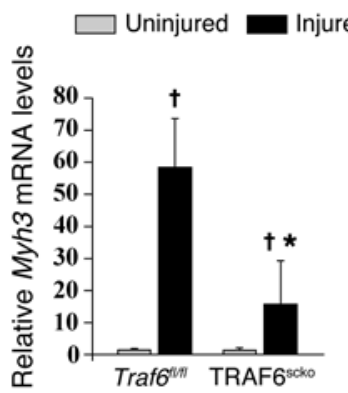

$\mathbf{F}$

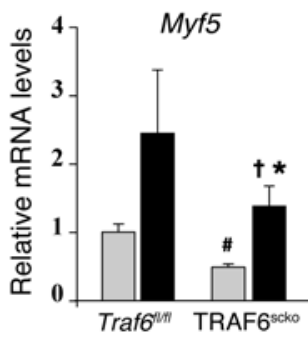

$\square$ Uninjured $\square$ Injured

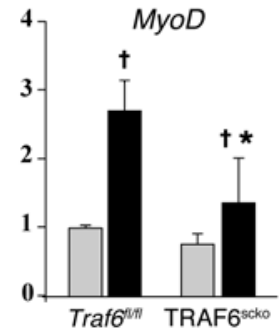

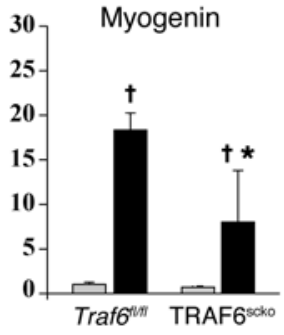

Figure 3. Deletion of Traf6 in satellite cells inhibits formation of new myofibers and expression of MRFs following injury. (A) Representative photomicrographs of days 0,3 , and 5 injured TA muscle section of Traf $6^{f / f f}$ and TRAFG $6^{\text {scko }}$ mice after immunostaining for eMyHC. Nuclei were identified by staining with DAPI. Scale bars: $20 \mu \mathrm{m}$. (B) Quantification of number of eMyHC+ fibers per field $\left(\sim 0.15 \mathrm{~mm}^{2}\right)$ in $5 \mathrm{~d}$ injured TA muscle of Traf6 $6^{f / f l}$ and TRAFG ${ }^{\text {scko }}$ mice. (C) Average CSA of eMyHC+ fibers in 5d injured TA muscle. (D) Representative immunoblots demonstrating the protein levels of eMyHC and unrelated protein GAPDH in TA muscle of Traff fl/fl and TRAFG ${ }^{\text {scko }}$ mice at various time point after BaCl, injection. (E and F) Relative mRNA levels of Myh3 (E) and Myf5, Myod, and myogenin (F) 3d after injury. Error bars represent SD. $n=5-6$ in each group. ${ }^{*} P<0.05$ (vs. injured TA muscle of Traf $6^{f / f f l}$ mice) by unpaired $t$ test. ${ }^{\dagger} P<0.05$ (vs. corresponding uninjured TA muscle of TrafG $\sigma^{f / f l}$ or TRAFG ${ }^{\text {scko }}$ mice) by paired $t$ test. ${ }^{~} P<0.05$ (vs. uninjured TA muscle of Traff $6^{f / f l}$ mice) by paired $t$ test.

matic increase in TRAF6 protein levels compared with uninjured muscle. Intriguingly, a much less severe upregulation of TRAF6 protein was noticeable in the injured TA muscle of TRAF6 ${ }^{\text {scko }}$ mice (Figure 4D), suggesting that satellite cells are an important source of TRAF6 protein in injured skeletal muscle of mice.

To further clarify the role of TRAF6 in satellite cell homeostasis, we performed FACS analysis of satellite cell content. Consistent with above results, TA muscle of TRAF $6^{\text {scko }}$ demonstrated a modest upregulation in $\alpha 7$-integrin ${ }^{+}$cells following injury compared with that of corresponding Traf $6^{f / / l}$ mice (Supplemental Figure 3).

We also measured the transcript levels of a few target genes of Notch pathway, a known regulator of satellite cell dynamics in both quiescent and activated states (27-29). Notably, mRNA levels of Hey1, HeyL, Hes1, and Hes6 were significantly reduced in injured muscle of TRAF6 $6^{\text {scko }}$ mice (Supplemental Figure 4). It has been reported that HES1, HEY1, and HEYL are involved in maintaining the quiescent status of satellite cells (28). Interestingly, we found that the transcript levels of HeyL and Hes1 were diminished in uninjured muscle of TRAF6 $6^{\text {scko }}$ compared with uninjured muscle of Traf $6^{f / f l}$ mice (Supplemental Figure 4). These results further suggest that TRAF6 is essential for the maintenance of satellite stem cell pool and function in vivo.

TRAF6 is required for self-renewal of satellite cells. To understand the role of TRAF6 in self-renewal of satellite cells, we established single myofiber cultures from extensor digitorum longus (EDL) muscle of Traf6 $6^{f / f l}$ and TRAF6 $6^{\text {scko }}$ mice, and the myofiber-associated satellite cells were analyzed at 0 hours or 72 hours of culturing. Consistent with our in vivo results, a significant reduction in the number of quiescent $\mathrm{PAX}^{+}$satellite cells was clearly evident in freshly isolated fibers of TRAF6 $6^{\text {scko }}$ mice (Figure 5, A and B). 
A
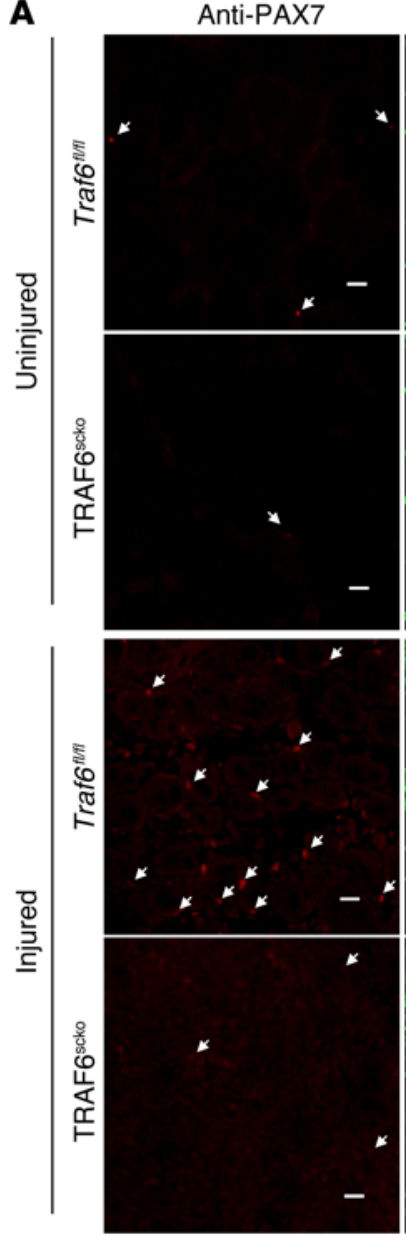

B

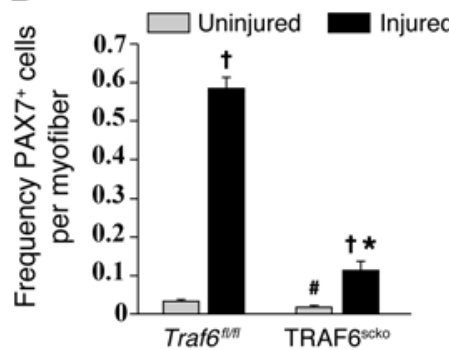

Anti-laminin
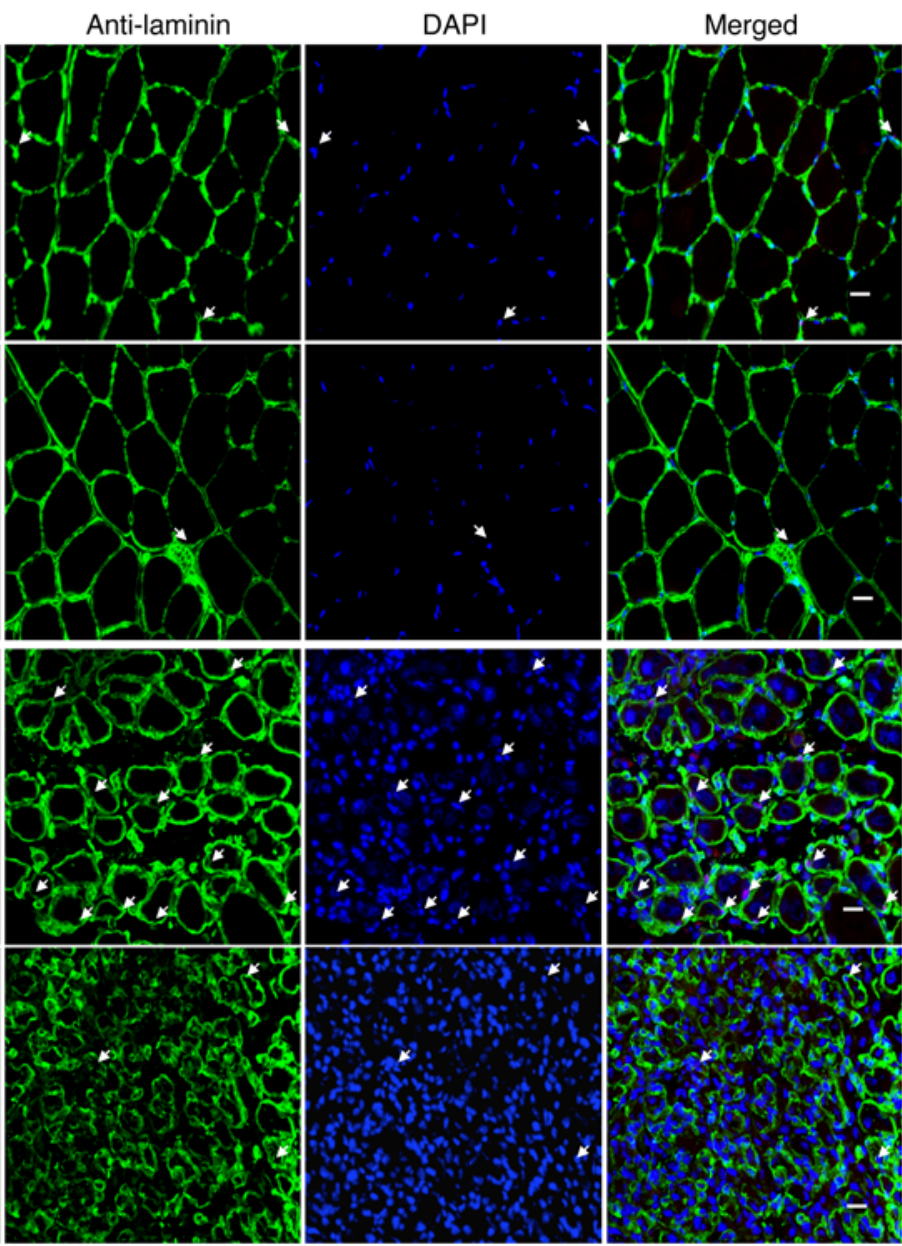

C



D



Figure 4. TRAF6 is required for maintenance of satellite cell pool in skeletal muscle. (A) Representative individual and merged photomicrographs of TA muscle transverse sections stained for PAX7 and laminin of uninjured and 5d-injured Traf6 ${ }^{f / f f}$ and TRAFG ${ }^{\text {scko }}$ mice. Nuclei were identified by costaining with DAPI. Arrows point to PAX7+ cells. Scale bars: $20 \mu \mathrm{m}$. (B) Quantification of average number of PAX7+ cells per myofiber in uninjured and injured TA muscle of Traf6 ${ }^{f / f l}$ and TRAFG ${ }^{\text {scko }}$ mice. (C) Relative mRNA of Pax7 in uninjured and 3d-injured TA muscle of Traf6 $6^{f / f l}$ and TRAFG ${ }^{\text {scko }}$ mice assayed by performing qPCR. (D) Protein levels of PAX7, TRAF6, and unrelated protein GAPDH in uninjured and $5 \mathrm{~d}$ injured TA muscle of Traf6 $f^{\text {fl/fl}}$ and TRAF6 ${ }^{\text {scko }}$ mice. Error bars represent SD. ${ }^{\dagger} P<0.05$ (vs. corresponding uninjured TA muscle of Traf6 $f^{f / f f}$ or TRAFG ${ }^{\text {scko }}$ mice) by unpaired $t$ test. $n=4$ in each group for $\mathbf{A}-\mathbf{C}$. ${ }^{*} P<0.05$ (vs. injured TA muscle of Traff $f^{f / f l}$ mice) by unpaired $t$ test. ${ }^{\#} P<0.05$ (vs. uninjured TA muscle of Traf $6^{f / f f}$ mice) by unpaired $t$ test. U, uninjured; I, injured.

Intriguingly, the majority of $\mathrm{PAX}^{+}$cells associated with TRAF6 6 scko myofibers were also expressing MyoD (Figure 5, A and C). Corresponding fibers from Traf $6^{f / f l}$ mice contained approximately 5-6 satellite cells that were predominantly $\mathrm{PAX}^{+} / \mathrm{MyoD}^{-}$(Figure 5, B and $\mathrm{C}$ ). The reduced number of $\mathrm{PAX} 7^{+}$satellite cells on freshly isolated myofibers of TRAF $6^{\text {scko }}$ mice and the untimely expression of MyoD implies a premature inclination of TRAF6-null satellite cells toward entering the cell cycle and failure to self-renew. To further understand the myogenic potential of TRAF6-deficient satellite cells, myofiber-associated cells were fixed after 72 hours in suspension culture and immunostained for PAX7 and MyoD. Myofiberassociated $\mathrm{Traf6}^{\mathrm{fl} / \mathrm{fl}}$ satellite cells displayed a normal activation profile characterized by formation of satellite cell clusters and normal distribution of self-renewing $\left(\mathrm{PAX}^{+} / \mathrm{MyoD}^{-}\right)$, proliferating $\left(\mathrm{PAX}^{+} / \mathrm{MyoD}^{+}\right)$, and differentiating $\left(\mathrm{PAX7}^{-} / \mathrm{MyoD}^{+}\right)$cells. However, myofibers from TRAF $6^{\text {scko }}$ mice exhibited scattered distribu- 
A
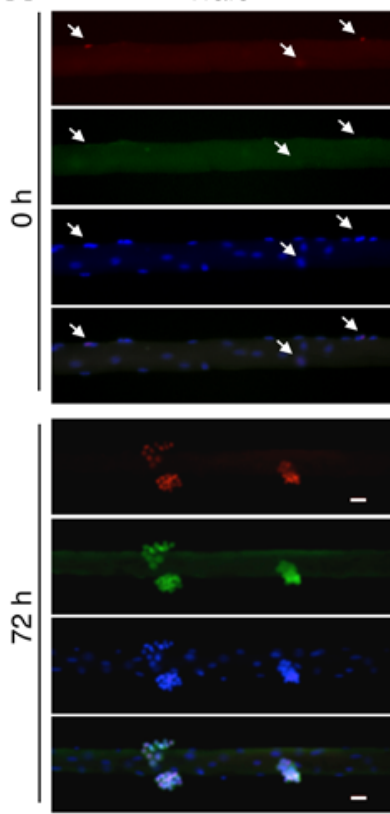

$F$
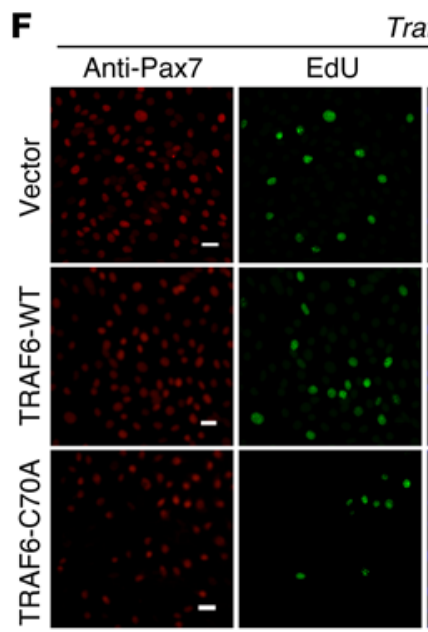

TRAF6 ${ }^{\text {scko }}$
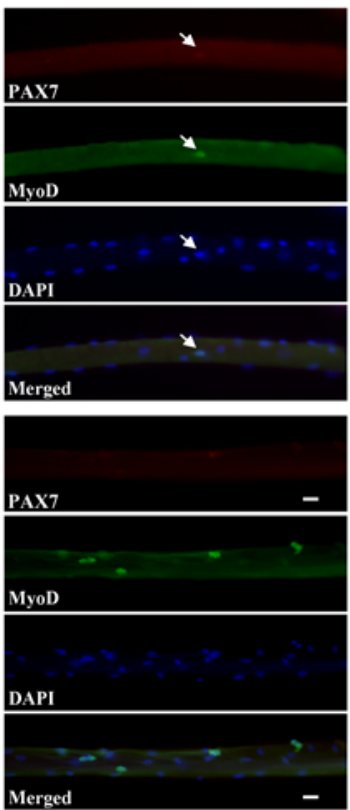

Traf6 $^{+/+}$
B

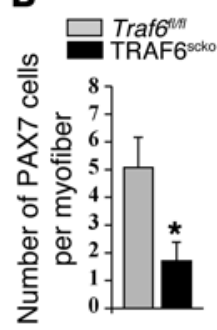

C

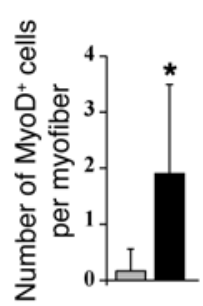

D



E

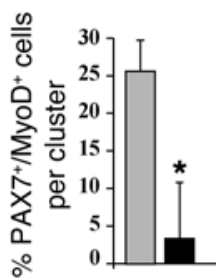

G

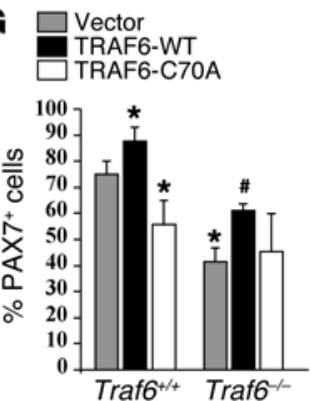

H

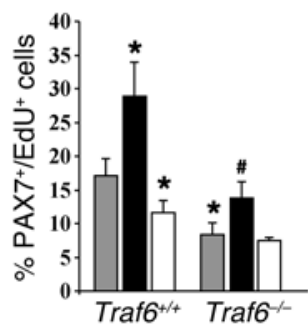



Figure 5. Deletion of Traf6 in satellite cells leads to premature differentiation. Single myofibers were isolated from EDL muscle of Traf6 ${ }^{f / f l}$ and TRAFG $^{\text {scko }}$ mice. Immediately after isolation or after $72 \mathrm{~h}$ of culturing, myofibers were collected and stained for PAX7 and MyoD. Nuclei were counterstained with DAPI. (A) Representative individual and merged images of freshly isolated and $72 \mathrm{~h}$ cultured myofibers from Traf6 ${ }^{f / f l}$ and TRAF6 ${ }^{\text {scko }}$ mice stained with PAX7, MyoD, and DAPI. Scale bars: $20 \mu \mathrm{m}$. (B and $\mathbf{C}$ ) Quantification of number of PAX7 $7^{+}(\mathbf{B})$ and MyoD $\mathrm{D}^{+}$(C) cells per myofiber immediately after isolation. Quantification of (D) number of cells per cluster on each myofiber, and (E) percentage of self-renewing (i.e., $\left.P A X 7^{+} / M_{y o D^{-}}\right)$cells per myofiber following $72 \mathrm{~h}$ of culturing. Analysis was done using 20-25 myofibers for each mouse at each time point. $n=3$ mice in each group for $\mathbf{A}-\mathbf{E}$. (F) $\mathrm{Traf6}^{+/+}$and $\mathrm{Traf6}^{-/-}$myogenic cultures were transfected with vector alone (pcDNA3) or plasmids expressing TRAF6-WT or TRAF6C70A cDNA. After $72 \mathrm{~h}$, the cells were pulse-labeled with EdU for 90 minutes and analyzed for the expression of Pax7 protein and EdU incorporation. Nuclei were identified by staining with DAPI. Scale bar: $20 \mu \mathrm{m}$. (G-H) Quantification of percentage of PAX7 $7^{+}$cells (G), and PAX7 $7^{+} /$EdU ${ }^{+}$cells (H) in cultures transfected with vector alone, TRAF6-WT, or TRAF6C70A cDNA. $n=4$ in each group. Error bars represent SD. ${ }^{*} P<0.05$ (vs. Traf6 $6^{f / f l}$ myofiber cultures or Traf6 ${ }^{+/+}$ cultures transfected with vector alone) by unpaired $t$ test (for $\mathbf{B}-\mathbf{E}$ ) and paired $t$ test (for $\mathbf{G}$ and $\mathbf{H}$ ). ${ }^{\#} P<0.05$ (vs. corresponding Traf6 ${ }^{-/-}$cells transfected with vector alone) by paired $t$ test.

tion of singlet and couplet cells and no evident clusters originating from a single clone (Figure 5A). Moreover, the majority of cells were $\mathrm{PAX7}^{-} / \mathrm{MyoD}^{+}$, and a few cells that were $\mathrm{PAX}^{+}$were unanimously $\mathrm{MyoD}^{+}$(Figure 5, D and E), suggesting that deletion of Traf6 inhibits self-renewal and causes precocious differentiation.

TRAF6 was initially identified as an adaptor protein. However, recent studies have shown that it also functions as an E3 ubiquitin ligase, catalyzing K63-linked autoubiquitination as well as ubiquitination of target proteins, which stimulates protein-protein interaction $(12,13,30,31)$. Mutation of cysteine residue to alanine (C7OA) in the TRAF6 zinc finger domain renders it unable to interact and bind with the UBC13-UEV complex (12). The TRAF6C70A mutant has previously been used to study the role of TRAF6 E3 ubiquitin ligase activity in various cellular responses $(13,19,30)$. To understand the role of E3 ubiquitin ligase activity of TRAF6, we investigated the effects of over- 
A

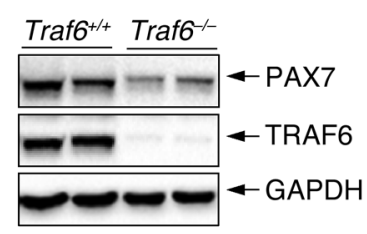

B



E

Traf6 $^{+/+}$Traf6 $^{-1}$

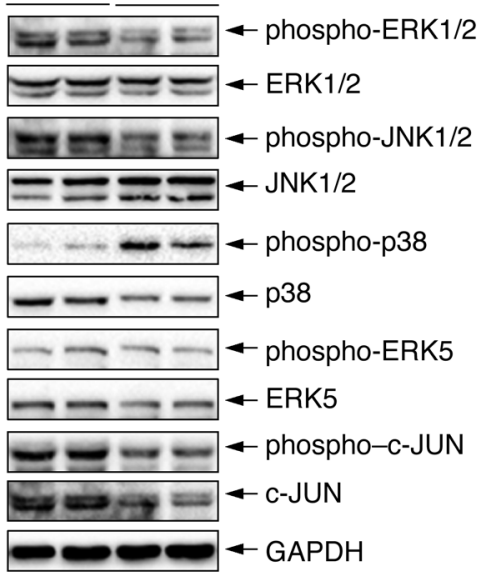

C
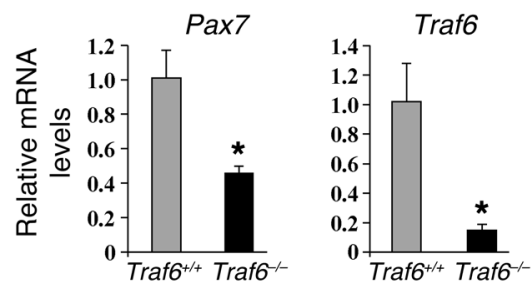

D
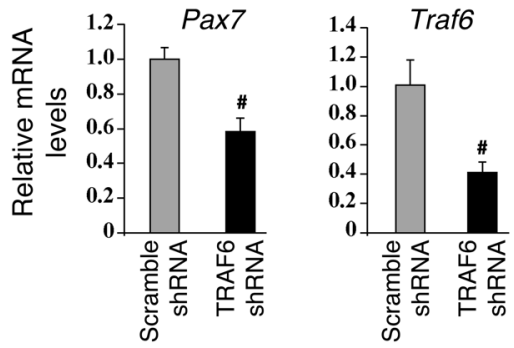

$\mathbf{F}$
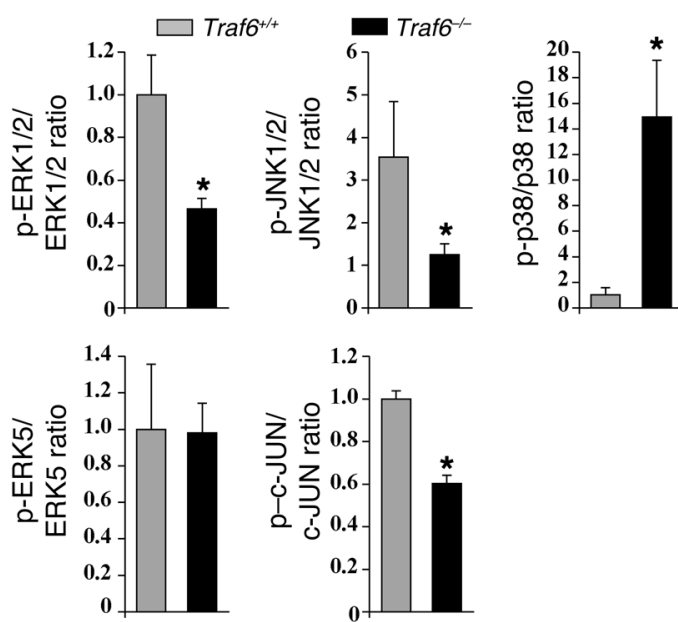

G

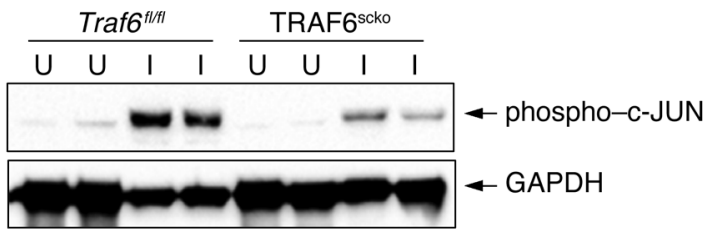

Figure 6. TRAF6 regulates PAX7 expression and activation of MAPKs in myogenic cells. (A) Immunoblots demonstrate protein levels of PAX7 and TRAF6 in Traf6 ${ }^{+/+}$and Traf6 $^{-/-}$cultures. (B) Densitometry quantification of relative protein levels of PAX7 and TRAF6 in Traf6 $6^{+/}$and Traf6 $6^{-/-}$cultures. $n=3$ in each group. (C) Relative mRNA levels of Pax7 and Traf6 in Traf6 ${ }^{+/+}$and Traf6 ${ }^{-/-}$cultures assayed by performing qPCR. $n=4$ in each group. (D) Primary myoblasts prepared from WT mice were transfected with scrambled shRNA or TRAF6 shRNA, and the mRNA levels of Pax7 and Traf6 were measured by performing qPCR. (E) Representative immunoblots from 2 independent experiments demonstrate the levels of phosphorylated and total ERK1/2, JNK1/2, p38, ERK5, and c-JUN in Traf6 $6^{+/}$and Traf6 $/ /$cultures. (F) Densitometry quantification of levels of phosphorylated and total ERK1/2, JNK1/2, p38, ERK5, and c-JUN in Traf6 ${ }^{+/+}$and Traf6 ${ }^{--}$cultures. $n=4-5$ in each group. (C) Immunoblots demonstrate the levels of phosphorylated c-JUN and an unrelated protein GAPDH in uninjured and injured TA muscle of Traf6 $6^{f / f l}$ and TRAFG ${ }^{\text {scko }}$ mice. Error bars represent SD. ${ }^{*} P<0.05$ (vs. Traf6 ${ }^{+/+}$cul- $^{-}$ tures) by unpaired $t$ test. ${ }^{\#} P<0.05$ (vs. cultures transfected with scrambled shRNA) by unpaired $t$ test. $\mathrm{U}$, uninjured; I, injured.

expression of WT TRAF6 (i.e., TRAF6-WT) and TRAF6C70A mutant on proliferation and self-renewal of satellite cells. Our attempts to directly isolate primary myoblasts from skeletal muscle of tamoxifen-treated TRAF $6^{\text {sko }}$ mice were unsuccessful due to reduced number of myofiber-associated satellite cells in these mice and failure of the cells to grow in culture upon seeding. To circumvent this problem, we isolated satellite cells from hind limb muscle of nontamoxifen-treated TRAF $6^{\text {scko }}$ mice and expanded them in culture, followed by treatment with vehicle alone (i.e., $\mathrm{Traf}^{+/+}$cells) or 4-hydroxytamoxifen to induce tamoxifen-mediated Cre recombination (henceforth $\mathrm{Traf6}^{-1-}$ cells). Both $\mathrm{Traf6}^{+/+}$and Traf6 $6^{-/}$myogenic cells were transfected with vector alone (pcDNA3), TRAF6-WT, or TRAF6C70A plasmids. After 72 hours of transfection, cellular proliferation was evaluated by pulse labeling the cells with 5-Ethynyl-2'-deoxyuridine (EdU). The cultures were also stained with anti-Pax7 (Figure $5 F)$. Interestingly, overexpression of TRAF6-WT protein significantly increased the number of $\mathrm{PAX} 7^{+}$cells or $\mathrm{PAX}^{+} / \mathrm{EdU}^{+}$cells, whereas overexpression of TRAF6C70A significantly reduced them compared with those transfected with vector alone in 
A

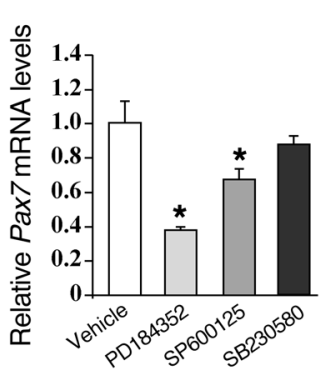

B

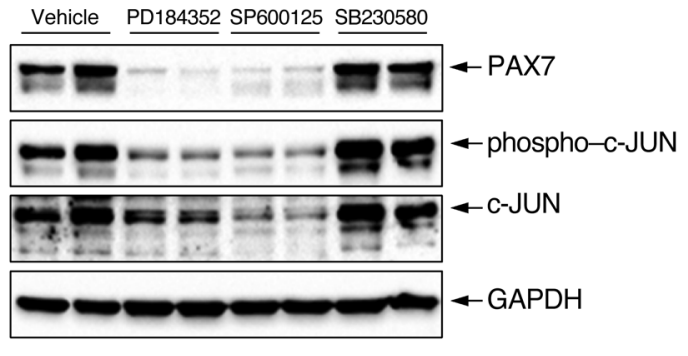

D
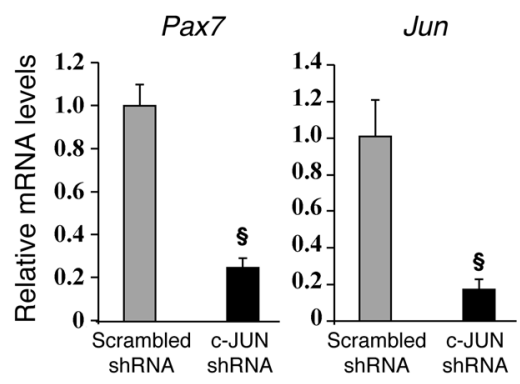

$\mathbf{F}$

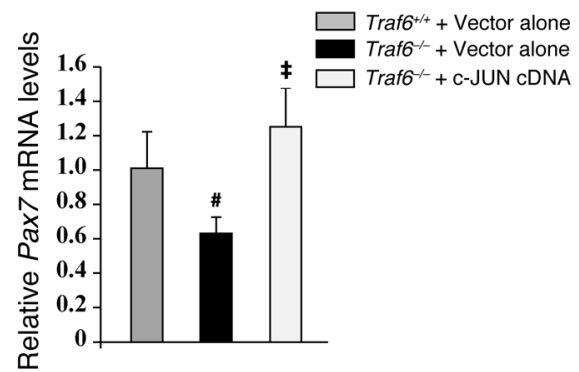

E

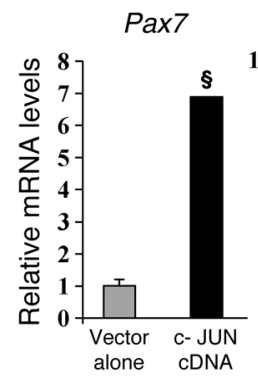

C
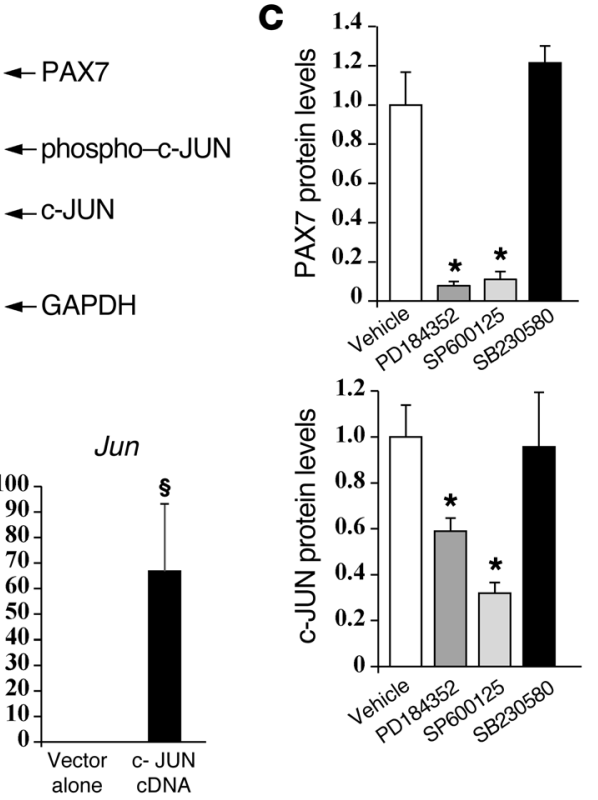

$\mathbf{G}$

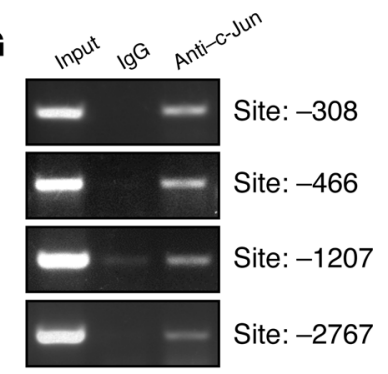

H


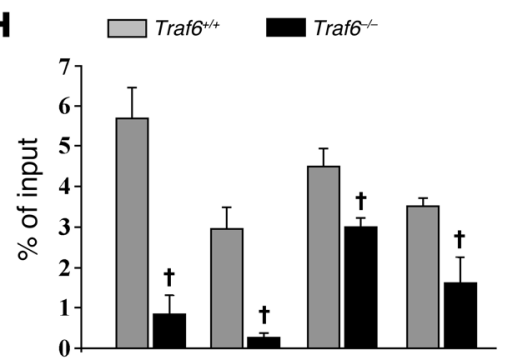

Figure 7. Role of MAPK signaling in gene expression of PAX7 in satellite cells. Primary myoblasts prepared from WT mice were treated with vehicle alone, $2 \mu \mathrm{M}$ PD184352, $20 \mu \mathrm{M}$ SP600125, or $20 \mu \mathrm{M}$ SB230580 for 4h. (A) Relative mRNA levels of Pax7 mRNA. (B) Representative immunoblots from 2 independent experiments demonstrate the levels of PAX7 and phosphorylated and total c-JUN protein. (C) Densitometry quantification of PAX7 and phosphorylated and total c-JUN protein after treatment with various inhibitors. $n=4$ in each group. (D) Relative mRNA levels of Pax7 and Jun after $72 \mathrm{~h}$ of transfection of myoblasts with scrambled or c-JUN shRNA construct. (E) Relative mRNA levels of Pax7 and Jun in primary myoblasts after $72 \mathrm{~h}$ of transfection with vector alone or c-JUN cDNA. (F) Relative mRNA levels of Pax7 in Traf6 ${ }^{-1-}$ cultures upon transfection with c-JUN cDNA. $n=4$ in each group. (G) Primary myoblasts were processed for ChIP assay for the binding of c-JUN at its putative consensus DNA sequence in Pax7 promoter. Agarose gel images of semi-quantitative RT-PCR demonstrate enrichment of Jun at indicated c-JUN/AP1 sites in mouse Pax7 promoter. The numbers indicate the position of consensus sequence upstream of first ATC in exon 1 of the Pax7 gene. (H) ChIP assay followed by qPCR analysis depicting percentage of input enrichment of c-JUN at specific sites in Pax7 promoter in Traf6 $6^{+/+}$and Traf6 ${ }^{-/-}$cultures. $n=4$ in each group. Error bars represent SD from mean. ${ }^{*} P<0.05$ (vs. cultures treated with vehicle alone) by paired $t$ test. ${ }^{\circledR} P<0.05$ (vs. corresponding cultures transfected with scrambled shRNA or vector alone) by unpaired $t$ test. ${ }^{\#} P<0.05$ (vs. Traf6 ${ }^{+/+}$cultures transfected with vector alone) by paired $t$ test. ${ }^{\ddagger} P<0.05$ (vs. Traf6 ${ }^{-/-}$cells transfected with vector alone) by paired $t$ test. ${ }^{\dagger} P<0.05$ (vs. rrafG $^{-/ \text {c }}$ cultures) by unpaired $t$ test.

$\mathrm{Traf6}^{+/+}$myogenic cultures (Figure $5, \mathrm{~F}-\mathrm{H}$ ). Number of $\mathrm{PAX}^{+}$or $\mathrm{PAX}^{+} / \mathrm{EdU}^{+}$cells was significantly reduced in Traf6 ${ }^{--}$cultures compared with $\mathrm{Traf}^{+/+}$cultures transfected with vector alone. While overexpression of TRAF6-WT significantly improved the number of $\mathrm{Pax}^{+} / \mathrm{EdU}^{+}$cells in Traf6 ${ }^{--}$cultures, there was no increase by overexpression of TRAF6C70A (Figure 5, F-H). By performing immunostaining for PAX7 and MyoD, we also investigated whether TRAF6 affects the proportion of PAX7-/MyoD ${ }^{+}$ cells in myogenic cultures. Results showed that the proportion of PAX7 $-\mathrm{MyoD}^{+}$cells was significantly higher in Traf $^{-/-}$cultures compared with Traf6 ${ }^{+/+}$cultures. Overexpression of TRAF6-WT significantly reduced the proportion of $\mathrm{PAX7}-/ \mathrm{MyoD}^{+}$cells in both $\mathrm{Traf6}^{+/+}$and $\mathrm{Traf6}^{-/-}$cultures. By contrast, overexpression of TRAF6C70A mutant significantly increased the proportion of $\mathrm{PAX7}^{-} / \mathrm{MyoD}^{+}$cells in Traf6 $6^{+/+}$cultures, but it had no significant effect in $\mathrm{Traf6}^{-}$cultures (Supplemental Figure 5, A and B). Increased expression of TRAF6-WT or TRAF6C70A protein in transfected cultures was confirmed by performing Western blot (Supplemental Figure 5C). Collectively, these results suggest that TRAF6 E3 ubiquitin ligase activity is essential for self-renewal, as well as proliferation and prevention of precocious differentiation of satellite cells.

TRAF6 is required for the expression of $P A X 7$ and the activation of MAPK signaling pathway in satellite cells. We next sought to determine whether TRAF6 is required for the expression of PAX7 in satellite cells. Our Western blot analysis confirmed that treatment 
A

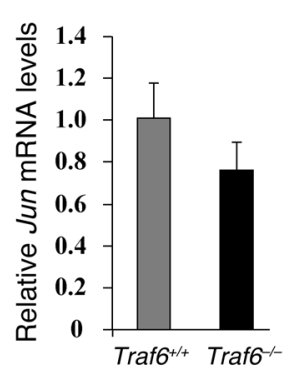

B

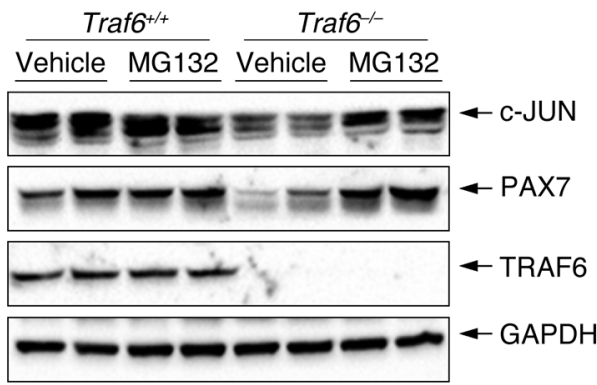

C

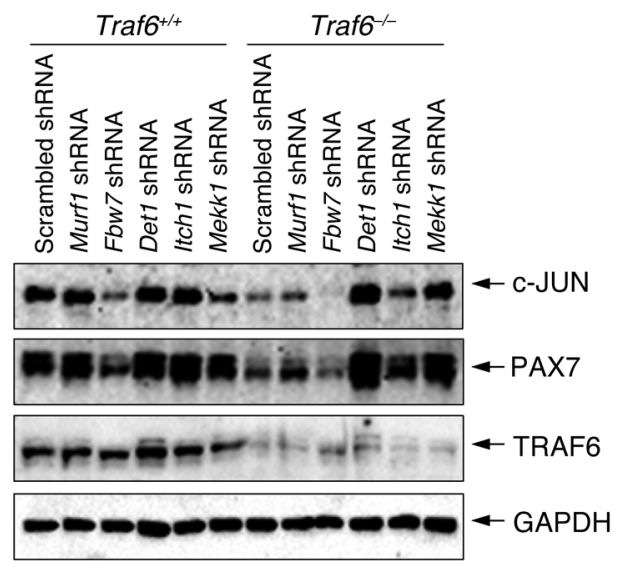

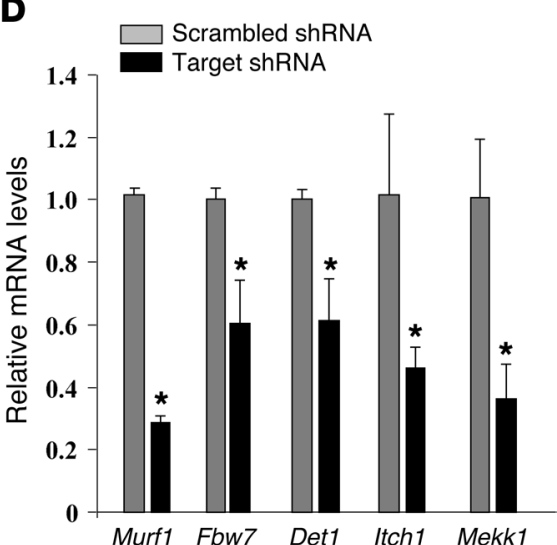

Figure 8. Role of TRAF6 in stabilization of c-JUN protein in satellite cells. (A) Relative mRNA levels of Jun in Traf6 $6^{+/+}$and Traf6 ${ }^{-/-}$myogenic cultures assayed by performing qPCR. (B) Traf6 $\sigma^{+/+}$and Traf6 ${ }^{-/-}$cells were treated with vehicle alone or $3 \mu \mathrm{M} \mathrm{MG132}$ (a proteasome inhibitor) for $12 \mathrm{~h}$, and the protein levels of c-JUN, PAX7, TRAF6 and unrelated protein GAPDH were measured by Western blot. Representative immunoblots from 2 independent experiments. (C) Traf6 ${ }^{+/+}$and Traf6 $^{-/-}$cells were transfected with scrambled shRNA or that targeting Murf1, Fbw7, Det1, Itch1, or Mekk1 mRNA for 72h, and the cells were processed to measure protein levels of c-JUN, PAX7, and TRAF6. Immunoblots demonstrate the levels of c-JUN, PAX7, TRAF6, and unrelated protein GAPDH. (D) Relative mRNA levels of Murf1, Fbw7, Det1, Itch1, and Mekk1 after transfection of WT cultures with their respective shRNA constructs. $n=3$ in each group. Error bars represent SD. ${ }^{*} P<0.05$ (vs. scrambled shRNA-transfected cultures) by unpaired $t$ test.

of TRAF6 $6^{\text {scko }}$ myoblast cultures with 4-hydroxytamoxifen (i.e., $\mathrm{Traf6}^{-/-}$cells) drastically reduces the protein levels of TRAF6 (Figure $6, \mathrm{~A}$ and $\mathrm{B})$. Consistent with our in vivo results, a drastic reduction in both protein and mRNA levels of Pax7 was noticeable in $\mathrm{Traf6}^{-/}$cultures compared with $\mathrm{Traf6}^{+/+}$cultures (Figure 6, A-C). Moreover, shRNA-mediated knockdown of TRAF6 significantly reduced the mRNA levels of Pax 7 in primary myogenic cultures from WT mice (Figure 6D). Given that PAX7 is a transcription factor, we also investigated whether PAX7 regulates the levels of TRAF6 in myogenic cells. However, no significant difference was noticeable in the levels of TRAF6 upon shRNA-mediated knockdown or overexpression of PAX7 in cultured myogenic cells from WT mice (Supplemental Figure 6).

Since TRAF6 is an important upstream regulator of MAPK, $\mathrm{AKT}$, and NF- $\mathrm{B}$ signaling pathways, we next sought to investigate whether TRAF6 has a role in the activation of these signaling pathways in satellite cells. Results showed that the phosphorylation levels of ERK1/2 and JNK1/2 were considerably reduced, while no such effect was observed on the phosphorylation of ERK5 in Traf6 $^{-/-}$cells compared with Traf6 ${ }^{+/+}$cells (Figure 6, E and F). Intriguingly, ablation of TRAF6 increased the levels of phosphorylated p38 MAPK (Figure 6, E and F), aligning with a role of p38 in promoting progression through the myogenic lineage $(32,33)$. Remarkably, we found that phosphorylated and total protein levels of c-JUN, a direct phosphorylation target of ERK1/2 and JNK, were also significantly diminished as a result of deletion of Traf6 (Figure 6, E and F). Furthermore, our Western blot analysis showed that in vivo ablation of Traf6 in satellite cells (i.e., tamoxifen-treated TRAF6 $6^{\text {scko }}$ vs. Traf $6^{f / f l}$ mice) also reduced the levels of phosphorylated c-JUN protein in $5 \mathrm{~d}$-injured skeletal muscle of mice (Figure 6G).

TRAF6 E3 ubiquitin ligase is essential for the AKT membrane localization and phosphorylation in response to growth stimuli $(13,34)$. However, we found that there was no significant difference in phosphorylation or total levels of AKT or its downstream phosphorylation targets, such as GSK-3 $\beta$, mTOR, and p70S6K (Supplemental Figure 7, A and B) or Akt kinase activity (Supplemental Figure 7, C and D) between $\mathrm{Traf6}^{+/+}$and $\mathrm{Traf6}^{-/-}$cells. Since TRAF6 mediates the activation of NF- $\kappa$ B in response to several cytokines and microbial products (11-14), we next investigated whether TRAF 6 is required for the activation of NF- $\kappa$ B in satellite cells. The DNA-binding activity of NF- $\mathrm{BB}$ was comparable between $\mathrm{Traf6}^{+/+}$and Traf6 $6^{-/-}$cells (Supplemental Figure 8A). Furthermore, there was no significant difference in the levels of 

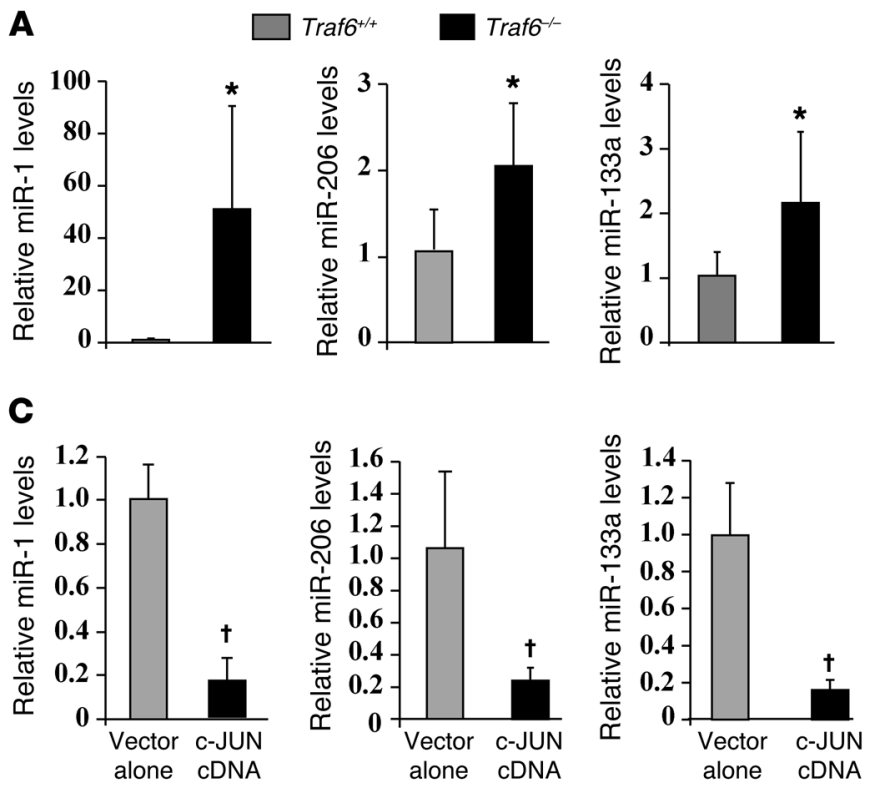

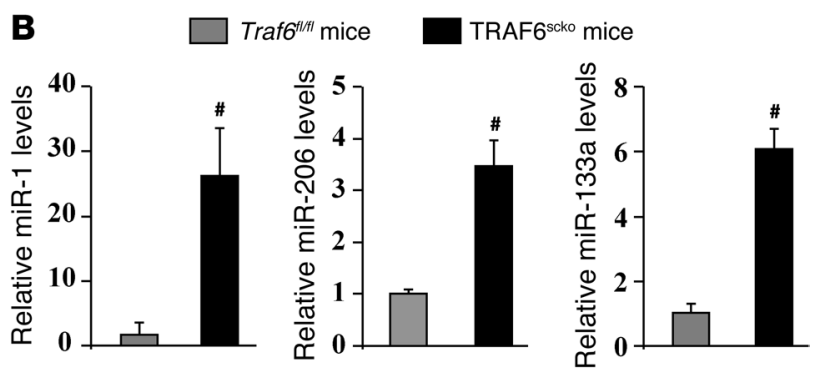

Figure 9. Role of TRAF6 in the expression of MyomiRs in satellite cells. (A) Relative levels of miR-1, miR-206, and miR-133a in Traf6 $6^{+/+}$and Traf6 $6^{-/-}$cultures. (B) TA muscle of adult Traff $6^{f / f l}$ and TRAFG ${ }^{\text {scko }}$ mice were injected with $100 \mu \mathrm{l} 1.2 \% \mathrm{BaCl}_{2}$ solution, and 5 days later, muscles were isolated and relative levels of miR-1, miR-206, and miR-133a were measured by qPCR. (C) Primary myoblasts prepared from WT mice were transfected with vector alone (pcDNA3) or c-JUN cDNA, and after 72h, the levels of miR-1, miR-206, and miR-133a were measured by performing qPCR assay. $n=4$ per group for all the experiments here. ${ }^{*} P<0.05$ (vs. TrafG ${ }^{+/+}$cultures). ${ }^{*} P<0.01$ (vs. injured TA muscle of Traff $f^{f / f l}$ mice) by unpaired $t$ test. ${ }^{\dagger} P<0.01$ (vs. cultures transfected with vector alone) by unpaired $t$ test.

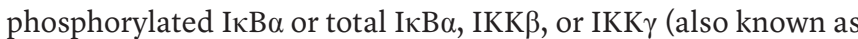
NEMO) between $\mathrm{Traf6}^{+/+}$and $\mathrm{Traf6}^{-/-}$myogenic cultures (Supplemental Figure 8, B and C). We also measured in vitro kinase activity of IKK $\beta$ and found no difference between Traf $^{+/+}$and $\mathrm{Traf6}^{-/}$cultures (Supplemental Figure 8D). In another experiment, we measured NF-B reporter gene activity by transfecting the cells with pNF-кB-Luc plasmid. Interestingly, we found a small but significant increase in the reporter gene activity in Traf6 $^{-/}$cultures compared with Traf6 $^{+/+}$cultures (Supplemental Figure $8 \mathrm{E}$ ). Moreover, a significant increase in NF- $\kappa \mathrm{B}$ activity was also noticeable in WT myogenic cultures in which TRAF6 was knocked down using shRNA technique (Supplemental Figure $8 \mathrm{~F}$ ). Taken together, these results suggest that TRAF6 specifically regulates MAPKs and has no major effect on the activation of AKT or NF- $\kappa \mathrm{B}$ signaling in satellite cells in naive conditions.

ERK1/2 and JNK1/2 regulate the expression of PAX7 through c-JUN transcription factor. To understand the direct role of MAPK signaling pathways in PAX7 expression, primary myoblasts prepared from WT mice were treated with specific MAPK inhibitors: PD184352, which inhibits ERK1/2; SP600125, which inhibits JNK1/2; and SB203580, which inhibits the p38MAPK pathway. Interestingly, cells treated with ERK1/2 or JNK1/2 inhibitors showed significantly reduced mRNA and protein levels of PAX7, while those treated with p38 inhibitor had no effect on PAX7 levels (Figure 7, A-C). Intriguingly, we also found that the cells treated with ERK1/2 and JNK inhibitors (but not with p38 inhibitor) exhibited a decrease in phosphorylated and total c-JUN levels (Figure 7, B and C). We next investigated whether ERK1/2 and JNK1/2 pathways are required for the proliferation and self-renewal of satellite cells. By performing Western blot, we confirmed that ERK1/2 and JNK1/2 are phosphorylated in both quiescent and cultured activated satellite cells (Supplemental Figure 9A). Using single myofiber cultures, we examined the effects of inhibition of ERK1/2 or JNK1/2 on self-renewal of satellite cells. Results showed that treatment with PD184352 or SP600125 for 72 hours drastically reduced the number of cells per cluster and number of $\mathrm{PAX}^{+} / \mathrm{MyoD}^{-}$cells on myofibers (Supplemental Figure 9, B-D). These results demonstrate that both ERK1/2 and JNK1/2 pathways are involved in the self-renewal and proliferation of satellite cells.

To understand the explicit role of c-JUN in regulation of PAX7 expression, we performed loss-of-function and gain-offunction experiments. In agreement with the preceding findings, shRNA-mediated knockdown of c-JUN sharply reduced the mRNA levels of Pax7 in primary myoblast cultures from WT mice (Figure 7D). By contrast, overexpression of c-JUN significantly increased the mRNA levels of Pax7 in myoblast cultures (Figure 7E). Moreover, overexpression of c-JUN restored Pax7 levels in $\mathrm{Traf6}^{-/-}$cultures (Figure 7F). Since c-JUN possesses a transactivation domain, we contemplated that c-JUN regulates PAX7 expression through direct binding to $\operatorname{Pax} 7$ promoter. Our in silico analysis of an approximately $3.5-\mathrm{kb}$ region upstream of the transcription start site in Pax7 promoter revealed multiple potential consensus sequences for the c-JUN/AP1 transcription factor. To understand whether c-JUN enriches the Pax7 promoter, we performed ChIP assay. Intriguingly, we found high enrichment of c-JUN in Pax7 promoter at 4 consensus sites in myogenic cultures from WT mice (Figure 7G). We next studied the potential involvement of these sites in TRAF6-mediated regulation of Pax7. Quantitative PCR (qPCR) revealed that the 
A

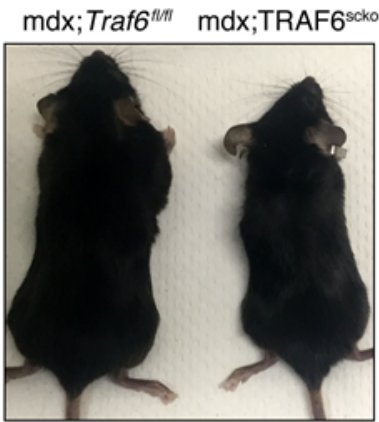

B

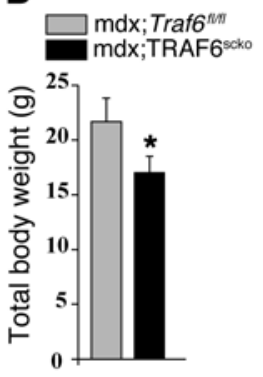

C

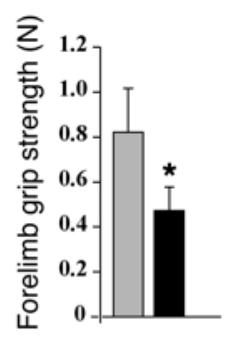

D

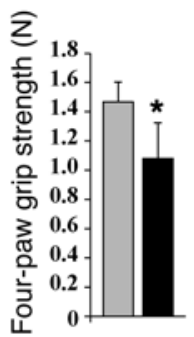

E





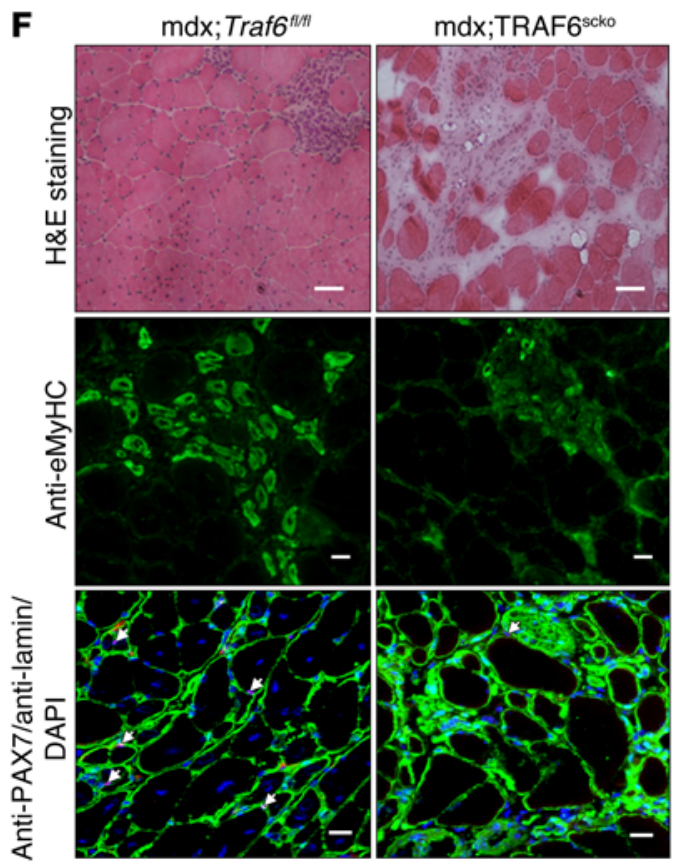

G

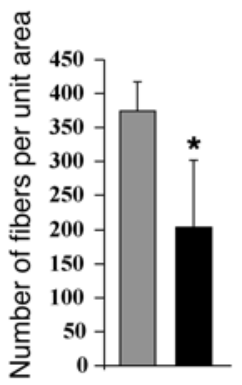

I

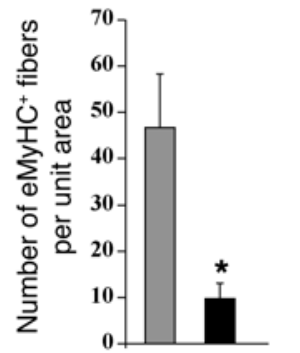

H

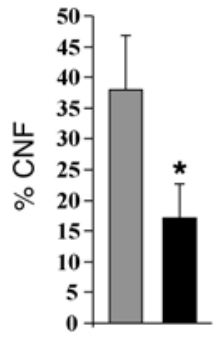

J



Figure 10. Depletion of TRAF6 in satellite cells exacerbates myopathy in mdx mice. (A) Representative pictures of 8-week-old mdx;Traf $6^{f / f f l}$ and mdx;TRAFG ${ }^{\text {scko }}$ mice. (B) Average body weight. (C) Forelimb and total grip strength normalized with body weight. (D) Average wet weight of TA, GA, and quadriceps (Quad) muscle of $\mathrm{mdx}$; Traf6 $6^{f / f l}$ and $\mathrm{mdx}$; TRAFG ${ }^{\text {scko }}$ mice. (E) CK activity in serum of 8-week-old Traf6 ${ }^{f / / f l}$ and TRAF6 ${ }^{\text {scko }}$ mice. (F) Representative photomicrograph of GA muscle sections of 8-week-old mdx;Traff fl/fl and mdx;TRAF6 scko mice after staining for H\&E, anti-eMyHC, or anti-PAX7/anti-laminin/DAPI. Arrows indicate satellite cells in muscle sections. Scale bars: $50 \mu \mathrm{m}$ for H\&E images, $20 \mu \mathrm{m}$ for eMyHC and PAX7 images. (G-J) Quantification of number of fibers per unit area $(\mathbf{G})$, percentage of centrally nucleated fibers $(\mathbf{H})$, number of eMyHC fibers per unit area $(\mathbf{I})$, and percentage of PAX7+ cells/ fiber (J). Error bars represent SD. $n=4$ or 5 mice in each group. ${ }^{*} P<0.05$ (vs. corresponding mdx; Traf $6^{f / f l}$ mice) by unpaired $t$ test.

fold enrichment (over input) of c-JUN to specific c-JUN sites in Pax7 promoter was significantly diminished in Traf6 ${ }^{-1-}$ cultures compared with $\mathrm{Traf6}^{+/+}$cultures (Figure $7 \mathrm{H}$ ). Collectively, these results suggest that TRAF6-mediated signaling augments PAX7 levels through the activation of ERK1/2 and JNK1/2, which in turn activate c-JUN transcription that directly interacts with the promoter region to enhance the gene expression of Pax7.

A recent study has demonstrated that the activation of NF- $\mathrm{KB}$ increases the number of PAX7 cells in mouse models of cancer cachexia (35). We investigated whether forced activation of NF- $\mathrm{KB}$ can improve the levels of PAX7 in TRAF6-deficient cells. Traf $6^{+/+}$and $\mathrm{Traf}^{-/}$cultures were transfected with vector alone or a constitutive active (ca) mutant of IKK $\beta$ (i.e., IKK-2 S177E S181E), and the cells were analyzed 72 hours later by Western blot or immunostaining. We found that overexpression of
caIKK $\beta$ had no effect on the protein levels of PAX7 in $\mathrm{Traf}^{+/+}$or Traf6 $^{-/}$cultures (Supplemental Figure 10A). Moreover, overexpression of caIKK $\beta$ did not affect the proliferation of $\mathrm{PAX}^{+}$cells in $\mathrm{Traf6}^{+/+}$or Traf6 $6^{-/}$cultures measured by EdU incorporation (Supplemental Figure 10, B and C). Furthermore, overexpression of caIKK $\beta$ had no significant effect on the number of $\mathrm{PAX}^{+}$or $\mathrm{PAX7}^{-} / \mathrm{MyoD}^{+}$cells in $\mathrm{Traf6}^{+/+}$or Traf6 $6^{-/}$cultures (Supplemental Figure 10, D-F). While NF- $\mathrm{kB}$ may have an important function in augmenting the proliferation of satellite cells and inhibiting their terminal differentiation in pathological conditions such as cancer cachexia (35), it does not affect self-renewal or proliferation of satellite cells in naive conditions.

TRAF6 prevents proteolytic degradation of c-JUN in satellite cells. Our preceding results showed that not only phosphorylation, but total levels of c-JUN are also reduced in $\mathrm{Traf}^{-/-} \mathrm{cul}-$ 
A

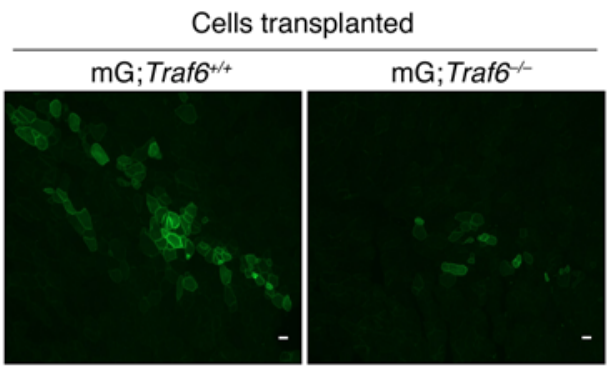

B

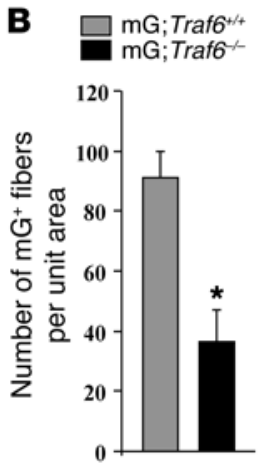

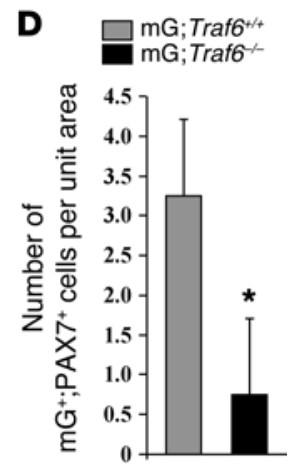

C

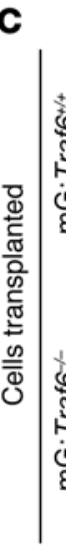

Anti-PAX7

$\mathrm{mG}$

DAPI

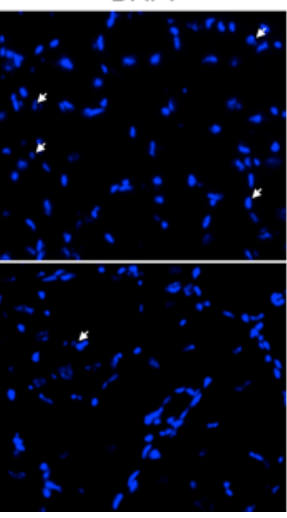

Merged

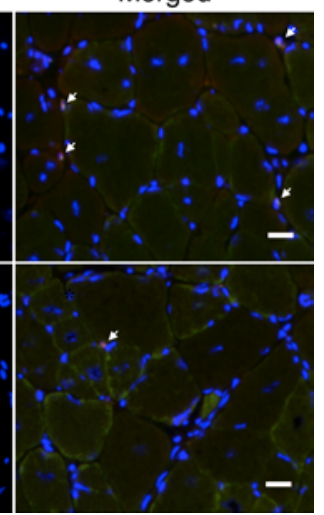

Figure 11. TRAF6 is required for satellite cell proliferation and self-renewal following transplantation in dystrophic muscle. TA muscle of 8-weekold mdx mice was injured by i.m. injection of $100 \mu \mathrm{l} 1.2 \% \mathrm{BaCl}_{2}$ solution. After $24 \mathrm{~h}$, the muscle was injected with $1 \times 10^{6} \mathrm{mG} ; \operatorname{Traf6}^{+/+}$or mG; Traf6 $6^{-/-}$cells. After 28 days, the TA muscle was isolated and processed for histological analysis. (A) Representative photomicrograph demonstrating mG-labeled fibers originating from $\mathrm{mG} ; \mathrm{TrafG}^{+/+}$and $\mathrm{mG} ; \mathrm{TrafG}^{-/-}$cells in TA muscle sections of mdx mice. Scale bars: $20 \mu \mathrm{m}$. (B) Quantification of number of mG ${ }^{+}$ fibers in TA muscle of mdx mice. (C) Self-renewal of $\mathrm{mG}$; Traf6 ${ }^{+/+}$and $\mathrm{mG} ;$ Traf6 $^{-/-}$satellite cells was analyzed by immunostaining with PAX7 and DAPI. (D) Quantification of number of $\mathrm{mG}^{+} ; \mathrm{PAX7}^{+}$cells. Error bars represent SD. $n=4$ in each group. ${ }^{*} P<0.05$ (vs. TA muscle of mdx mice transplanted with $\mathrm{mG} ; \operatorname{TrafG}^{+/+}$cells) by unpaired $t$ test.

tures. Recent studies have shown that c-JUN undergoes ubiquitination-dependent proteolytic degradation in many conditions (36-41). We investigated whether loss of TRAF6 reduces c-JUN protein levels through repression of its gene expression or through enhancing proteasome-mediated degradation in satellite cells. We first measured mRNA levels by performing qPCR assay. While there was a small decrease in the mRNA levels of Jun in $\mathrm{Traf6}^{-/-}$cells compared with $\mathrm{Traf6}^{+/+}$cells, the effect was not statistically significant (Figure 8A). To understand the role of proteasome, we treated cultured $\mathrm{Traf}^{+/+}$and $\mathrm{Traf6}^{-/-}$cells with proteasome inhibitor MG132 for 12 hours, and the levels of c-JUN protein were measured by Western blot. Interestingly, treatment with MG132 considerably improved the levels of c-JUN and PAX7 proteins in $\mathrm{Traf6}^{-/-}$cultures (Figure $8 \mathrm{~B}$ ).

A number of E3 ubiquitin ligases such as MEKK1, FBW7, DCX ${ }^{\text {hDET1-hCOP1 }}$ ITCH, and MuRF1 have now been identified, which cause K48-linked ubiquitination of c-JUN, leading to its proteolytic degradation (36-41). We investigated whether loss of TRAF 6 perturbs the activity of any of these ubiquitin ligases, leading to enhanced degradation of c-JUN. Traf6 $6^{+/+}$and Traf6 $^{-/-}$ cells were transfected with plasmid expressing scrambled, Mekk1, Fbw7, Det1, Itch, or Murf1 shRNA for 72 hours, and the protein levels of c-JUN and PAX7 were measured by performing Western blot. Interestingly, shRNA-mediated knockdown of Itch, Det1, and Mekk1 considerably improved the levels of c-JUN, as well as PAX7, in $\mathrm{Traf6}^{-/-}$cultures (Figure 8C). Surprisingly, we found that knockdown of FBW7 reduced the protein levels of c-JUN and PAX7 in both $\mathrm{Traf6}^{+/+}$and $\mathrm{Traf6}^{-/}$cultures. There was no effect of knockdown of Murf1 on c-JUN or PAX7 protein levels (Figure $8 \mathrm{C}$ ). By performing qPCR analysis, we confirmed that transfection with specific shRNAs significantly reduced the levels of their target mRNA in WT myogenic cultures (Figure 8D). Collectively, these results suggest that loss of TRAF6 leads to the aberrant regulation of multiple E3 ubiquitin ligases, which causes the degradation of c-JUN protein through ubiquitin-proteasome system in satellite cells.

Ablation of TRAF6 augments the levels of muscle-specific microRNAs in satellite cells. MicroRNAs are a class of short (19-22 bp) noncoding RNAs that function as posttranscriptional regulators of gene expression. Recently, a group of muscle-specific miRNAs (MyomiRs) - consisting of miR-1, miR-206, miR-133a, and miR$133 \mathrm{~b}$ - has been shown to be critical in regulating skeletal muscle development (42-44). Expression levels of miR-1, miR-206, and miR-133 have been found to be sharply upregulated during myo- 
genesis (44). Furthermore, miR-1 and miR-206 have been shown to promote satellite cell differentiation through direct targeting of Pax 7 mRNA (45). Strikingly, we found that the levels of miR-1, miR-206, and miR-133a were increased in Traf6 ${ }^{-1-}$ cells compared with $\mathrm{Traf6}^{+/+}$cells (Figure 9A). Moreover, the levels of miR-1, miR-206, and miR-133a were also found to be significantly upregulated in injured TA muscle of TRAF $6^{\text {scko }}$ mice compared with corresponding injured muscle of Traf $6^{f / f l}$ mice (Figure 9B). Since our preceding findings draw a possible role of c-JUN in mediating TRAF6 regulation of Pax7 transcription, we further delineated whether TRAF6-mediated regulation of myomiRs is also brought about through a c-Jun-dependent mechanism. Interestingly, overexpression of c-JUN repressed the levels of miR-1, miR-206, and miR-133a in cultured satellite cells (Figure 9C). These results are suggestive of a possible posttranscriptional role of TRAF6/MAP$\mathrm{K} / \mathrm{c}$-JUN/AP1 axis in mediating PAX7 expression.

Deletion of Traf6 in satellite cells blunts regeneration and exacerbates myopathy in mdx mice. Functional inactivation of the dystrophin gene is the primary cause of Duchenne muscular dystrophy in humans and in mdx mice (46). Dystrophic muscle undergoes repetitive rounds of degeneration and regeneration $(46,47)$. To further clarify the role of TRAF6-mediated signaling in satellite cells, we crossed TRAF $6^{\text {scko }}$ mice with mdx mice to generate mdx;TRAF $6^{\text {scko }}$ and littermate mdx;Traf $6^{f / f l}$ mice. At the age of 3 weeks, the mice were treated with tamoxifen to induce Cre-mediated recombination, and analysis was conducted at the age of 8 weeks. Deletion of TRAF6 led to a significant reduction in the size and body weight of mdx;TRAF6 $6^{\text {scko }}$ mice compared with littermate $\mathrm{mdx} ; \operatorname{Traf} 6^{f l / f l}$ mice (Figure 10, A and B). Furthermore, mdx;TRAF6 $6^{\text {scko }}$ mice displayed a significant reduction in forelimb and total 4-paw grip strength (Figure 10C). Following a treadmill-based exercise tolerance protocol $(48,49)$, we also compared exercise capacity. Results showed that mdx;TRAF $6^{\text {scko }}$ mice performed poorly in all the parameters such as total running time, maximum speed, distance, work performed, and power compared with littermate mdx;Traf $6^{f / f l}$ mice (Supplemental Figure 11). Our results also showed that wet weight of gastrocnemius (GA) and quadriceps muscles was significantly reduced, whereas levels of creatine kinase (CK) in plasma were significantly increased in mdx;TRAF $6^{\text {scko }}$ mice compared with littermate mdx; Traf $6^{f l / f l}$ mice (Figure 10, D and E). Analysis of H\&E-stained GA muscle sections showed signs of exacerbated myopathy marked by increased necrotic areas and overall decrease in total number of myofibers and proportion of CNF in GA muscle of mdx;TRAF6 ${ }^{\text {scko }}$ mice compared with mdx; Traf6 ${ }^{f / f l}$ mice (Figure 10, F-H). Similar results were obtained in quadriceps and TA muscles and diaphragm (Supplemental Figure 12). Moreover, compared with mdx; Traf6 $6^{f / f l}$ mice, the number of $\mathrm{eMyHC}^{+}$myofibers was dramatically reduced in GA muscle of mdx;TRAF ${ }^{\text {scko }}$ mice, which further suggests that myofiber regeneration is repressed in these mice (Figure 10, F and I). Furthermore, the number of $\mathrm{PAX}^{+}$cells was significantly reduced in GA muscle of mdx;TRAF6 $6^{\text {scko }}$ mice compared with littermate $\mathrm{mdx}$;Traf $6^{f l / f l}$ mice (Figure 10, $\mathrm{F}$ and J). Taken together, these results suggest that satellite cell-specific ablation of TRAF6 exaggerates myopathy in mdx mice due to impairment in muscle regeneration.
Deletion of Traf6 in satellite cells inhibits their engraftment in dystrophic muscle of $m d x$ mice. To further confirm the role of TRAF6 in satellite cell self-renewal and regenerative myogenesis, we also investigated whether ablation of TRAF6 inhibits their engraftment in skeletal muscle. We crossed TRAF6 $6^{\text {scko }}$ mice with membrane-targeted tandem dimer Tomato/membrane-targeted green fluorescent protein $(\mathrm{mT} / \mathrm{mG})$ reporter mice and generated mTmG Traf6 $6^{\text {flfl }}$ Pax7-CreER and control mTmG WT Pax7-CreER mice. These mice express $\mathrm{mT}$ in naive conditions and $\mathrm{mG}$ after Cre-mediated excision (50). Satellite cells were isolated and purified, followed by treatment with tamoxifen for 48 hours, to generate $\mathrm{mG} ; \mathrm{Traf6}^{+/+}$and $\mathrm{mG} ; \operatorname{Traf6}^{-/-}$cells. TA muscle of $\mathrm{mdx}$ mice was given i.m. injection of $1.2 \% \mathrm{BaCl}_{2}$ solution for 24 hours followed by injection of $\mathrm{mG} ; \operatorname{Traf6}^{+/+}$or $\mathrm{mG} ; \operatorname{Traf6}^{-/}$satellite cells. After 4 weeks, the TA muscle was isolated, and transverse sections made were visualized directly for $\mathrm{mG}$ florescence (green) using a fluorescent microscope. Results showed that mG;Traf6 ${ }^{-/}$ cells had dramatically reduced engraftment capacity compared with $\mathrm{mG} ;$ Traf $^{+/+}$cells in dystrophic muscle of mdx mice (Figure 11, A and B). We also performed staining with anti-PAX7 to understand the fate of transplanted satellite cells following engraftment. Interestingly, the number of $\mathrm{PAX} 7 / \mathrm{mG}$ double-positive cells was significantly reduced in TA muscle of $\mathrm{mdx}$ mice transplanted with $\mathrm{mG} ; \mathrm{Traf6}^{-/}$cells compared with those transplanted with mG; Traf $6^{+/+}$cells (Figure 11, C and D). These results demonstrate that TRAF6-deficient satellite cells exhibit poor engraftment and fail to repopulate the satellite cell niche following transplantation into dystrophic muscle.

\section{Discussion}

Skeletal muscle regeneration involves activation of a complex array of signaling pathways not only in myogenic precursor cells, but also in damaged myofibers. Our present findings and previously published report (20) also highlight that a single signaling module (i.e., TRAF6) can have distinct roles when activated in muscle progenitor cells versus differentiated myofibers during regenerative myogenesis. Depletion of TRAF6 in myofibers augments regeneration potentially through mitigating inflammatory response (20). Interestingly, inhibition of TRAF6 in myofibers also improves Notch signaling in satellite cells in a non-cell-autonomous manner, leading to improvement in regeneration (20). In contrast, the results of the present investigation demonstrate that TRAF6 activity is critical for maintaining the pool of satellite cells and their function during regenerative myogenesis.

Failure of TRAF6-null satellite cells to drive proper regeneration could be attributed to failure in (i) activation and exiting quiescence, (ii) proper activation and entry to the cell cycle but a defect in one or more of the stages of myogenesis, or (iii) untimely activation and progression through the myogenic lineage. Reduced number of $\mathrm{PAX}^{+}$satellite cells in sham muscle (Figure 4) and freshly isolated myofibers of TRAF $6^{\text {scko }}$ mice accompanied with MyoD expression (Figure 5, A and B) excludes a possible activation defect, as reduced levels of PAX7 indicate loss of quiescence and upregulated MyoD levels imply premature activation $(1,4,7)$. Furthermore, lack of cell cluster formation on cultured myofibers from TRAF6 ${ }^{\text {scko }}$ mice suggests that TRAF6 is required for maintaining the replicative capacity of satellite cells (Figure 
5 , A and D). This inference is also supported by our findings that forced expression of TRAF6 in cultured satellite cells augments proliferation and increases the levels of PAX7 (Figure 5, F-H). Our systematic examination of the expression profile of various MRFs, and activation and proliferation dynamics of satellite cells, implicates loss of myogenic lineage upon deletion of TRAF6 due to repression of PAX7 expression, which in turn leads to precocious differentiation.

Our results further demonstrate that TRAF6 is an essential component of a signaling pathway that drives the gene expression of Pax7. TRAF6 regulates the levels of PAX7 in satellite cells through conditional activation and deactivation of specific MAPK signaling pathways (Figure 6). Increased expression of p38 MAPK in TRAF6-deficient cells (Figure 6, E and F) lends further support to the premise that loss of TRAF6 in myogenic cells leads to precocious differentiation. Signaling from $\mathrm{p} 38 \mathrm{MAPK}$ has been shown to play an essential role in regulating the later stages of myogenesis $(51,52)$. Quiescent satellite cells treated with p38 MAPK inhibitor fail to enter the cell cycle upon activation, whereas overexpression of $\mathrm{p} 38$ promotes exit from quiescence and leads to premature differentiation $(33,52)$. In contrast with $\mathrm{p} 38 \mathrm{MAPK}$, phosphorylation of ERK1/2 was reduced in TRAF6-deficient satellite cells (Figure 6, E and F), consistent with the role of ERK1/2 signaling in regulating the proliferation and self-renewal of satellite cells (53). Additionally, our results implicate a parallel role of JNK signaling pathway in regulation of satellite stem cell homeostasis. The levels of phosphorylated JNK1/2 protein were also reduced upon deletion of TRAF6 (Figure 6, E and F), and inhibition of JNK1/2 independently reduced the levels of PAX7, mirroring ERK1/2 inhibition (Figure 7, A-C). ERK1/2 and JNK1/2 signaling can have an antagonizing effect on the activation p38 MAPK, and vice versa $(54,55)$. Therefore, it is likely that the reduced ERK1/2 and JNK1/2 signaling brought about by deletion of TRAF6 leads to hyperactivation of p38 MAPK in satellite cells, which in turn promotes exit from quiescence and precocious differentiation.

The results of the present study also suggest that PAX7 regulation through ERK1/2 and JNK1/2 signaling takes place in a c-JUNdependent manner. TRAF6-null primary myogenic cells exhibited a reduction in total and phosphorylated levels of c-JUN protein (Figure 6, E and F), and inhibition of ERK1/2 or JNK1/2 similarly reduced levels of phosphorylated and total c-JUN in WT myoblasts (Figure 7, B and C). Our ChIP results further support the inference that c-JUN directly interacts with Pax7 promoter in satellite cells (Figure 7G), and this interaction is sustained through continued TRAF6-mediated signaling (Figure 7H). Surprisingly, we also discovered that TRAF6-mediated signaling prevents the degradation of c-JUN in satellite cells, potentially through suppressing the activity of specific E3 ubiquitin ligases such as DCX ${ }^{\mathrm{hDET1}-\mathrm{hCOP} 1}$, ITCH, and MEKK1 (Figure 8).

Our results also suggest a potential posttranscriptional role of c-JUN in modulating PAX7 levels. Pax7 mRNA has been identified as one of the direct regulatory targets of miR- 1 and miR-206 (45), both of which were upregulated in TRAF6-deficient satellite cells and injured muscle of TRAF $6^{\text {sko }}$ mice (Figure 9, A and B) and diminished upon overexpression of c-JUN (Figure 9C). The mechanisms by which TRAF6/c-JUN pathway reduces the levels of miR-1 and miR-206 in satellite cells remain enigmatic. Whether the alterations observed in miR-1 and miR-206 are mere phenotypic outcomes or possible auxiliary regulatory facets that modulate Pax7 expression in TRAF6 context requires further investigation.

The critical role of TRAF6 in regulating satellite cell function is also supported by our findings in $\mathrm{mdx}$ mice. Deletion of Traf6 in satellite cells of mdx mice led to exacerbated myopathy due to reduced number of satellite cells and impairment in regeneration of injured myofibers (Figure 10). Diminished myogenic potential of TRAF6-deficient satellite cells is also evident by our findings that these cells show poor engraftment upon transplantation into dystrophic muscle of mdx mice (Figure 11). Exhaustion of satellite cell myogenic capacity accompanies progression of dystrophinopathy, leading to cessation of muscle regeneration $(56,57)$. Although therapies aimed at improving the muscle microenvironment have been found to be transiently advantageous, extensive investigations have shown that premature loss of satellite cell function in dystrophic muscle is due to a cell autonomous defect $(4,56,57)$. Our present investigation provides initial evidence that modulating the levels of TRAF6 in satellite cells of dystrophic muscle or donor cells may prove to be beneficial in prolonging the myogenic potential and replenishing the reservoir of satellite cells.

In summary, the present study has identified a previously unrecognized role of TRAF6 in regulating satellite stem cell homeostasis and function. TRAF6-mediated regulation of satellite cells occurs through regulating the levels of PAX7. TRAF6 was shown to be necessary in maintaining the quiescent state of satellite cells under unchallenged conditions, sustaining the myogenic potential upon stimulation, and regulating self-renewal following activation.

\section{Methods}

Animals. TRAF6 $6^{\text {scko }}$ were generated by crossing Pax7-CreER mice (B6;129-Pax $7^{\mathrm{tm} 2.1(\mathrm{cre} / \mathrm{ERT} 2) \mathrm{Fan}} / \mathrm{J}$; The Jackson Laboratory) with $\operatorname{Traf6}^{\mathrm{f} / \mathrm{fl}}$ mice. Similarly, $\mathrm{mdx}$ mice were crossed with TRAF $6^{\text {sko }}$ to generate $\mathrm{mdx}$; TRAF6 $6^{\text {scko }}$ mice and littermate $\mathrm{mdx}$;Traf $6^{\text {fl/fl }}$ mice. TRAF $6^{\text {scko }}$ mice were

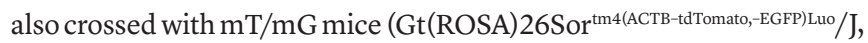
The Jackson Laboratory) for satellite cell transplantation experiment. Genotype of mice was determined by PCR from tail DNA. For deletion of Traf6, mice were i.p. injected with tamoxifen for 4 consecutive days and kept on tamoxifen-containing standard chow for the duration of the experiment. At the age of 8 weeks, $100 \mu \mathrm{l}$ of $1.2 \% \mathrm{BaCl}_{2}$ (SigmaAldrich) dissolved in saline was injected into the TA muscle of mice to induce necrotic injury.

Histological and morphometric analysis. H\&E staining of muscle sections and quantification of fiber CSA were performed as described in Supplemental Methods.

CK assay. Levels of CK in serum were determined as described in Supplemental Methods.

Isolation, culture, and staining of single myofibers. Single myofibers were isolated from EDL muscle after digestion with collagenase II (SigmaAldrich) and trituration as previously described $(20,58)$. Suspended fibers were cultured in 60-mm horse serum-coated plates in DMEM supplemented with 10\% FBS (Invitrogen), 2\% chicken embryo extract (Accurate Chemical and Scientific Corporation), $10 \mathrm{ng} / \mathrm{ml}$ basis fibroblast growth factor (PeproTech), and 1\% penicillin-streptomycin for 3 days. Freshly isolated fibers and cultured fibers were then fixed in $4 \%$ PFA and stained with anti-PAX7 (1:10, Developmental Studies Hybridoma Bank [DSHB]) and MyoD (1:200, sc-304, Santa Cruz Biotechnology Inc.). 
Satellite cell cultures. Satellite cells were isolated from the hind limbs of 8-week-old mice following a protocol as described (59). Traf6 ${ }^{-/}$cells were generated by treatment of TRAF $6^{\text {sko }}$ myogenic cells with $10 \mathrm{ng} / \mathrm{ml}$ 4-hydroxytamoxifen (Sigma-Aldrich) for 48 hours.

Immunofluorescence. For immunohistochemistry study, frozen TA muscle sections or paraformaldehyde-fixed myofiber or myoblast cultures were blocked in 1\% BSA in PBS for 1 hour and incubated with anti-Pax7 (1:10, DSHB), anti-eMyHC (1:200, clone F1.652, DSHB), anti-laminin (1:500, catalog L9393, Sigma-Aldrich), anti-MyoD (1:200, catalog sc-304, Santa Cruz Biotechnology Inc.), anti-TRAF6 (1:50, catalog sc-7221, Santa Cruz Biotechnology Inc.) in blocking solution at $4^{\circ} \mathrm{C}$ overnight under humidified conditions. The sections were washed briefly with PBS before incubation with Alexa Fluor 488 or 594-conjugated secondary antibody (1:3,000, Invitrogen) for 1 hour at room temperature and then washed 3 times for 15 minutes with PBS. The slides were mounted using fluorescence medium (Vector Laboratories) and visualized at room temperature on Nikon Eclipse TE 2000-U microscope, a digital camera (Nikon Digital Sight DS-Fi1), and Nikon NIS Elements BR 3.00 software. Image levels were equally adjusted using Abode Photoshop CS2 software.

Cell proliferation assay. Satellite cell proliferation was assayed by labeling the cells with EdU for 90 minutes using Click-iT EdU Cell Proliferation Assay kit (Invitrogen) as previously described (59).

shRNA and plasmid constructs. Various shRNAs and Pax7 cDNA were cloned in mammalian expression vectors, as described in Supplemental Methods.

Gene transfer by electroporation. To overexpress specific cDNA or shRNA in primary satellite cells, plasmid DNA was introduced into cells by electroporation (1,500 V, 10 ms for duration, 3 pulses) using the Neon transfection system following a protocol suggested by the manufacturer (Invitrogen).

EMSA. The method used for EMSA is described in Supplemental Methods.

ChIP assay. ChIP assay was performed following a method as previously described (60). In brief, primary myoblasts were crosslinked with $1 \%$ formaldehyde at room temperature for 10 minutes and washed with ice-cold PBS. Cross-linking was stopped by adding glycine to a final concentration of $0.125 \mathrm{M}$ for 5 minutes. After 2 washes with $0.05 \mathrm{~mm}$ PMSF in PBS, the cells were lysed in $10 \mathrm{~mm}$ Hepes $(\mathrm{pH}$ 8.0), $85 \mathrm{~mm} \mathrm{KCl}, 0.5 \%$ Igepal, and $2 \times$ protease inhibitor mixture. The nuclear fraction was collected by centrifugation and lysed for $20 \mathrm{~min}$ utes in $50 \mathrm{~mm}$ Tris- $\mathrm{HCl}, 10 \mathrm{~mm}$ EDTA, and $1 \%$ SDS at $4^{\circ} \mathrm{C}$. Nuclear extracts were sonicated (30 strokes at $30 \%$ for 15 seconds followed by 60 seconds of rest) while being kept in an ice-water bath. The lysate was cleared with salmon sperm DNA/protein G-agarose slurry (Cell Signaling Technology). Approximately $200 \mu \mathrm{l}$ of chromatin fraction was used for each immunoprecipitation assay. After a $\times 5$ dilution in ChIP dilution buffer, $20 \mu$ l were separated (input), and the remaining diluted chromatin was incubated with the indicated antibody against c-Jun (Santa Cruz Biotechnology) or rabbit IgG (negative control). Detection of the immunoprecipitated DNA was performed by PCR with primers targeting specific regions of the Pax7 promoter. The sequence of primers used for specific c-Jun sites upstream of first ATG in Exon 1 of Pax7 is described in Supplemental Table 1. For the quantification of fold enrichment, qPCR was performed and the percentage enrichment of input was reported.

In vitro kinase assay. The enzymatic activity of Akt and IKK were measured, as described in Supplemental Methods.
Western blot. Quantitative estimation of various proteins was performed by Western blot using a method as described $(18,61)$. Antibodies used were anti-TRAF6 (catalog 04-451, Millipore; catalog 597, MBL International; or catalog sc-7221, Santa Cruz Biotechnology), anti-Pax7 (DSHB), anti-MyoD (catalog sc-304, Santa Cruz Biotechnology Inc.), anti-eMyHC (clone F1.652, DSHB), anti-MyHC (clone MF20, DSHB), anti-phospho-c-JUN (catalog 3270, Cell Signaling Technology), anti-c-JUN (catalog sc-44, Santa Cruz Biotechnology), anti-phospho-ERK1/2 (catalog 9101, Cell Signaling Technology), anti-ERK1/2 (catalog 9102, Cell Signaling Technology), anti-phospho-JNK1/2 (catalog 9251, Cell Signaling Technology), anti-JNK1/2 (catalog 9252, Cell Signaling Technology), anti-phospho-p38 MAPK (catalog 9211, Cell Signaling Technology), anti-p38 MAPK (catalog 9212, Cell Signaling Technology), anti-phospho-ERK5 (catalog 3371, Cell Signaling Technology), antiERK5 (catalog 3372, Cell Signaling Technology), anti-phospho-AKT (catalog 4060, Cell Signaling Technology), anti-AKT (catalog 9272, Cell Signaling Technology), anti-phospho-GSK-3 $\beta$ (catalog 9327, Cell Signaling Technology), anti-GSK-3 $\beta$ (catalog 9315, Cell Signaling Technology), anti-phospho-mTOR (catalog 2974, Cell Signaling Technology), anti-mTOR (catalog 2972, Cell Signaling Technology), anti-phospho-p70S6K (catalog 9208, Cell Signaling Technology), anti-p70S6K (catalog 9202, Cell Signaling Technology), antiphospho-IкB $\alpha$ (catalog 2859, Cell Signaling Technology), anti-IкB $\alpha$ (catalog 9242, Cell Signaling Technology), anti-IKK $\beta$ (catalog 2684, Cell Signaling Technology), anti-IKK $\gamma$ (catalog 559675, BD Pharmingen), and anti-GAPDH (catalog 2118, Cell Signaling, Inc.). Antibodies were detected by chemiluminescence.

FACS. Satellite cells were analyzed by FACS as previously described $(20,58)$. Approximately $2 \times 10^{6}$ cells were incubated in DMEM (supplemented with 2\% FBS and 25 mM 4-[2-hydroxyethyl]-1piperazineethanesulfonic acid), and dead cells (positive for propidium iodide staining) were excluded from all FACS analyses. For satellite cell quantification or isolation from heterogeneous cell population, cells were immunostained with antibodies against CD45, CD31, Sca-1, and Ter-119 for negative selection (all PE conjugated, eBioscience), and with $\alpha 7$-integrin (MBL International) for positive selection. A tandem conjugate of R-PE (Alexa 647, Invitrogen) was used as a secondary antibody against $\alpha 7$-integrin. To detect TRAF6 expression in satellite cells, following the labeling with antibodies against CD45, CD31, Sca-1, Ter119 , and $\alpha 7$-integrin, the cells were fixed with $1 \%$ PFA (Sigma-Aldrich) and permeabilized using 0.2\% Triton X-100 (Thermo Fisher Scientific). The cells were then incubated with anti-TRAF6 (Santa Cruz Biotechnology Inc.) and Alexa 488 secondary antibody (Invitrogen). FACS analysis was performed on a C6 Accuri cytometer (BD Biosciences) equipped with 3 lasers. The output data was processed, and plots were prepared using FCS Express 4 RUO software (De Novo Software).

RNA isolation and qPCR assay. RNA isolation and qPCR for specific mRNA and miRs were performed using a method as described $(18,62)$. The sequence of the primers is described in Supplemental Table 1.

Grip strength measurements and exercise tolerance test. Muscle strength of mice was assayed by measuring grip strength and performing treadmill-based exercise tolerance tests, as described in Supplemental Methods.

Statistics. Results are expressed as mean \pm SD. Statistical analyses used Student's $t$ test to compare quantitative data populations with 
normal distribution and equal variance. A value of $P<0.05$ was considered statistically significant unless otherwise specified.

Study approval. All animal procedures were conducted in strict accordance with the institutional guidelines and were approved by the Institutional Animal Care and Use Committee and Institutional Biosafety Committee of the University of Louisville (IACUC, 13097).

\section{Author contributions}

SMH and AK designed research work, analyzed data, and wrote the manuscript. SMH conducted all the experiments and acquired data.

\section{Acknowledgments}

This work was supported by funding from NIH grants AR059810, AR068313, and AG029623 (to A. Kumar) and AG046950 (to S.M.
Hindi). We thank Yongwon Choi (University of Pennsylvania, Philadelphia, Pennsylvania, USA) for providing floxed Traf6 mice. We thank Shuichi Sato for his valuable help with exercise tolerance test, Sabrin Albeituni for help with FACS analysis, and Robert Miller for isolation of satellite cells by FACS method. We are grateful to Bryant G. Darnay (University of Texas MD Anderson Cancer Center, Houston, Texas, USA) for providing TRAF6C70A plasmid. We also thank Michael Rudnicki (University of Ottawa, Ottawa, Ontario, Canada) for his suggestions for our study.

Address correspondence to: Ashok Kumar, Department of Anatomical Sciences and Neurobiology, University of Louisville School of Medicine, 500 South Preston Street, Louisville, Kentucky 40202, USA. Phone: 502.852.1133; E-mail: ashok.kumar@louisville.edu.
1. Kuang S, Rudnicki MA. The emerging biology of satellite cells and their therapeutic potential. Trends Mol Med. 2008;14(2):82-91.

2. Relaix F, Marcelle C. Muscle stem cells. Curr Opin Cell Biol. 2009;21(6):748-753.

3. Relaix F, Zammit PS. Satellite cells are essentia for skeletal muscle regeneration: the cell on the edge returns centre stage. Development. 2012;139(16):2845-2856.

4. Yin H, Price F, Rudnicki MA. Satellite cells and the muscle stem cell niche. Physiol Rev. 2013;93(1):23-67.

5. Seale P, Sabourin LA, Girgis-Gabardo A, Mansouri A, Gruss P, Rudnicki MA. Pax7 is required for the specification of myogenic satellite cells. Cell. 2000;102(6):777-786.

6. Kuang S, Charge SB, Seale P, Huh M, Rudnicki MA. Distinct roles for Pax7 and Pax3 in adult regenerative myogenesis. J Cell Biol. 2006;172(1):103-113.

7. Kuang S, Kuroda K, Le Grand F, Rudnicki MA. Asymmetric self-renewal and commitment of satellite stem cells in muscle. Cell. 2007;129(5):999-1010.

8. Zammit PS, Golding JP, Nagata Y, Hudon V, Partridge TA, Beauchamp JR. Muscle satellite cells adopt divergent fates: a mechanism for self-renewal? J Cell Biol. 2004;166(3):347-357.

9. Olguin HC, Olwin BB. Pax-7 up-regulation inhibits myogenesis and cell cycle progression in satellite cells: a potential mechanism for self-renewal. Dev Biol. 2004;275(2):375-388.

10. von Maltzahn J, Jones AE, Parks RJ, Rudnicki MA. Pax7 is critical for the normal function of satellite cells in adult skeletal muscle. Proc Natl Acad Sci US A. 2013;110(41):16474-16479.

11. Chung JY, Lu M, Yin Q, Lin SC, Wu H. Molecular basis for the unique specificity of TRAF6. Adv Exp Med Biol. 2007;597:122-130.

12. Lamothe B, Besse A, Campos AD, Webster WK, Wu H, Darnay BG. Site-specific Lys-63-linked tumor necrosis factor receptor-associated factor 6 auto-ubiquitination is a critical determinant of IкB kinase activation. J Biol Chem. 2007;282(6):4102-4112.

13. Yang WL, et al. The E3 ligase TRAF6 regulates Akt ubiquitination and activation. Science. 2009;325(5944):1134-1138.

14. Inoue J, Gohda J, Akiyama T. Characteristics and biological functions of TRAF6. Adv Exp Med Biol. 2007;597:72-79.

15. Kobayashi T, et al. TRAF6 is a critical factor for dendritic cell maturation and development. Immunity. 2003;19(3):353-363.

16. Lomaga MA, et al. TRAF6 deficiency results in osteopetrosis and defective interleukin-1, CD40, and LPS signaling. Genes Dev. 1999;13(8):1015-1024.

17. Naito A, et al. Severe osteopetrosis, defective interleukin-1 signalling and lymph node organogenesis in TRAF6-deficient mice. Genes Cells. 1999;4(6):353-362

18. Paul PK, et al. Targeted ablation of TRAF6 inhibits skeletal muscle wasting in mice. J Cell Biol. 2010;191(7):1395-1411.

19. Paul PK, et al. The E3 ubiquitin ligase TRAF6 intercedes in starvation-induced skeletal muscle atrophy through multiple mechanisms. Mol Cell Biol. 2012;32(7):1248-1259.

20. Hindi SM, et al. Reciprocal interaction between TRAF6 and notch signaling regulates adult myofiber regeneration upon injury. Mol Cell Biol. 2012;32(23):4833-4845

21. Hindi SM, Sato S, Choi Y, Kumar A. Distinct roles of TRAF6 at early and late stages of muscle pathology in the mdx model of Duchenne muscular dystrophy. Hum Mol Genet. 2014;23(6):1492-1505.

22. Mueck T, et al. TRAF6 regulates proliferation and differentiation of skeletal myoblasts. Differentiation. 2011;81(2):99-106

23. Xiao F, Wang H, Fu X, Li Y, Wu Z. TRAF6 promotes myogenic differentiation via the TAK1/ p38 mitogen-activated protein kinase and Akt pathways. PLoS One. 2012;7(4):e34081.

24. Le Grand F, Rudnicki MA. Skeletal muscle satellite cells and adult myogenesis. Curr Opin Cell Biol. 2007;19(6):628-633.

25. Olguin HC, Yang Z, Tapscott SJ, Olwin BB. Reciprocal inhibition between Pax7 and muscle regulatory factors modulates myogenic cell fate determination. J Cell Biol. 2007;177(5):769-779.

26. Beauchamp JR, et al. Expression of CD34 and Myf5 defines the majority of quiescent adult skeletal muscle satellite cells. J Cell Biol. 2000;151(6):1221-1234.

27. Conboy IM, Rando TA. The regulation of Notch signaling controls satellite cell activation and cell fate determination in postnatal myogenesis. Dev Cell. 2002;3(3):397-409.

28. Bjornson CR, Cheung TH, Liu L, Tripathi PV, Steeper KM, Rando TA. Notch signaling is necessary to maintain quiescence in adult muscle stem cells. Stem Cells. 2012;30(2):232-242.

29. Mourikis P, Sambasivan R, Castel D, Rocheteau $\mathrm{P}$, Bizzarro V, Tajbakhsh S. A critical requirement for notch signaling in maintenance of the quiescent skeletal muscle stem cell state. Stem Cells. 2012;30(2):243-252.

30. Lamothe B, Webster WK, Gopinathan A, Besse A, Campos AD, Darnay BG. TRAF6 ubiquitin ligase is essential for RANKL signaling and osteoclast differentiation. Biochem Biophys Res Commun. 2007;359(4):1044-1049.

31. Deng L, et al. Activation of the IkappaB kinase complex by TRAF6 requires a dimeric ubiquitin-conjugating enzyme complex and a unique polyubiquitin chain. Cell. 2000;103(2):351-361.

32. Baeza-Raja B, Munoz-Canoves P. p38 MAPK-induced nuclear factor-kappaB activity is required for skeletal muscle differentiation: role of interleukin-6. Mol Biol Cell. 2004;15(4):2013-2026.

33. Troy A, Cadwallader AB, Fedorov Y, Tyner K, Tanaka KK, Olwin BB. Coordination of satellite cell activation and self-renewal by Par-complex -dependent asymmetric activation of $\mathrm{p} 38 \alpha / \beta$ MAPK. Cell Stem Cell. 2012;11(4):541-553.

34. Yang WL, Wu CY, Wu J, Lin HK. Regulation of Akt signaling activation by ubiquitination. Cell Cycle. 2010;9(3):487-497.

35. He WA, et al. NF-kB-mediated Pax7 dysregulation in the muscle microenvironment promotes cancer cachexia. J Clin Invest. 2013;123(11):4821-4835.

36. Li HH, et al. The ubiquitin ligase MuRF1 protects against cardiac ischemia/reperfusion injury by its proteasome-dependent degradation of phospho-c-Jun. Am J Pathol. 2011;178(3):1043-1058.

37. Wei W, Jin J, Schlisio S, Harper JW, Kaelin WG Jr. The v-Jun point mutation allows c-Jun to escape GSK3-dependent recognition and destruction by the Fbw7 ubiquitin ligase. Cancer Cell. 2005;8(1):25-33.

38. Xia Y, Wang J, Xu S, Johnson GL, Hunter T, Lu Z. MEKK1 mediates the ubiquitination and degradation of c-Jun in response to osmotic stress. $\mathrm{Mol}$ Cell Biol. 2007;27(2):510-517.

39. Babaei-Jadidi R, et al. FBXW7 influences murine 


\section{RESEARCH ARTICLE}

intestinal homeostasis and cancer, targeting Notch, Jun, and DEK for degradation. J Exp Med. 2011;208(2):295-312.

40. Wertz IE, et al. Human De-etiolated-1 regulates c-Jun by assembling a CUL4A ubiquitin ligase. Science. 2004;303(5662):1371-1374.

41. Gao M, et al. Jun turnover is controlled through JNK-dependent phosphorylation of the E3 ligase Itch. Science. 2004;306(5694):271-275.

42. Naguibneva I, et al. The microRNA miR-181 targets the homeobox protein Hox-A11 during mammalian myoblast differentiation. Nat Cell Biol. 2006;8(3):278-284.

43. Chen JF, et al. The role of microRNA-1 and microRNA-133 in skeletal muscle proliferation and differentiation. Nat Genet. 2006;38(2):228-233.

44. McCarthy JJ, Esser KA, Peterson CA, Dupont-Versteegden EE. Evidence of MyomiR network regulation of beta-myosin heavy chain gene expression during skeletal muscle atrophy. Physiol Genomics. 2009;39(3):219-226.

45. Chen JF, et al. microRNA-1 and microRNA-206 regulate skeletal muscle satellite cell proliferation and differentiation by repressing Pax7. J Cell Biol. 2010;190(5):867-879.

46. Hoffman EP, Brown RH Jr, Kunkel LM. Dystrophin: the protein product of the Duchenne muscular dystrophy locus. Cell. 1987;51(6):919-928.

47. Chakkalakal JV, Thompson J, Parks RJ, Jasmin BJ. Molecular, cellular, and pharmacological therapies for Duchenne/Becker muscular dystrophies. FASEB J. 2005;19(8):880-891.
48. Handschin C, et al. Skeletal muscle fiber-type switching, exercise intolerance, and myopathy in PGC-1 $\alpha$ muscle-specific knock-out animals. J Biol Chem. 2007;282(41):30014-30021.

49. Sato S, Ogura Y, Tajrishi MM, Kumar A. Elevated levels of TWEAK in skeletal muscle promote visceral obesity, insulin resistance, and metabolic dysfunction. FASEB J. 2015;29(3):988-1002.

50. Muzumdar MD, Tasic B, Miyamichi K, Li L, Luo L. A global double-fluorescent Cre reporter mouse. Genesis. 2007;45(9):593-605.

51. Bernet JD, Doles JD, Hall JK, Kelly Tanaka K, Carter TA, Olwin BB. p38 MAPK signaling underlies a cell-autonomous loss of stem cell self-renewal in skeletal muscle of aged mice. Nat Med. 2014;20(3):265-271.

52. Jones NC, et al. The $\mathrm{p} 38 \alpha / \beta$ MAPK functions as a molecular switch to activate the quiescent satellite cell. J Cell Biol. 2005;169(1):105-116.

53. Abou-Khalil R, et al. Autocrine and paracrine angiopoietin 1 /Tie- 2 signaling promotes muscle satellite cell self-renewal. Cell Stem Cell. 2009;5(3):298-309.

54. Munshi HG, et al. Differential regulation of membrane type 1-matrix metalloproteinase activity by ERK 1/2- and p38 MAPK-modulated tissue inhibitor of metalloproteinases 2 expression controls transforming growth factor-beta1-induced pericellular collagenolysis. J Biol Chem. 2004;279(37):39042-39050.

55. Boutros T, Chevet E, Metrakos P. Mitogen-activated protein (MAP) kinase/MAP kinase phos- phatase regulation: roles in cell growth, death, and cancer. Pharmacol Rev. 2008;60(3):261-310.

56. Tedesco FS, Dellavalle A, Diaz-Manera J, Messina G, Cossu G. Repairing skeletal muscle: regenerative potential of skeletal muscle stem cells. J Clin Invest. 2010;120(1):11-19.

57. Heslop L, Morgan JE, Partridge TA. Evidence for a myogenic stem cell that is exhausted in dystrophic muscle. JCell Sci. 2000;113(pt 12):2299-2308.

58. Dahiya S, et al. Elevated levels of active matrix metalloproteinase-9 cause hypertrophy in skeletal muscle of normal and dystrophin-deficient mdx mice. Hum Mol Genet. 2011;20(22):4345-4359.

59. Ogura Y, Mishra V, Hindi SM, Kuang S, Kumar A. Proinflammatory cytokine tumor necrosis factor (TNF)-like weak inducer of apoptosis (TWEAK) suppresses satellite cell self-renewal through inversely modulating Notch and NF-kB signaling pathways. J Biol Chem. 2013;288(49):35159-35169.

60. Tajrishi MM, Shin J, Hetman M, Kumar A. DNA methyltransferase $3 \mathrm{a}$ and mitogen-activated protein kinase signaling regulate the expression of fibroblast growth factor-inducible 14 (Fn14) during denervation-induced skeletal muscle atrophy. J Biol Chem. 2014;289(29):19985-19999.

61. Mittal A, et al. The TWEAK-Fn14 system is a critical regulator of denervation-induced skeletal muscle atrophy in mice. JCell Biol. 2010;188(6):833-849.

62. Liu W, et al. Hypoxia promotes satellite cell self-renewal and enhances the efficiency of myoblast transplantation. Development. 2012;139(16):2857-2865. 